Engineering Physics and Mathematics Division

Mathematical Sciences Section

\title{
A PROPOSAL FOR A USER-LEVEL, MESSAGE PASSING INTERfaCe IN A DISTRIBUTED MEMORY ENVIRONMENT
}

\author{
Jack J. Dongarra $\ddagger \S$ \\ Rolf Hempel थ \\ Anthony J. G. Hey $\dagger$ \\ David W. Walker $\S$ \\ $\ddagger$ Department of Computer Science \\ 107 Ayres Hall \\ Knoxville, TN 37996-1301 \\ $\dagger$ Department of Electronics and Computer Sciences \\ University of Southampton \\ Southampton, SO9 5NH \\ United Kingdom \\ I Gesellschaft für Mathematik und Datenverarbeitung mbH \\ P. O. Box 1316 \\ D-5205 Sankt Augustin 1 \\ Germany \\ $\S$ Mathematical Sciences Section \\ Oak Ridge National Laboratory \\ P.O. Box 2008, Bldg. 6012 \\ Oak Ridge, TN 37831-6367
}

Date Published: February 1993

Research was supported by the Applied Mathematical Sciences Research Program of the Office of Energy Research, U.S. Department of Energy, by the Defense Advanced Research Projects Agency under contract DAAL03-91-C-0047, administered by the Army Research Office, and by the Center for Research on Parallel Computing.

Prepared by the

Oak Ridge National Laboratory

Oak Ridge, Tennessee 37831

managed by

Martin Marietta Energy Systems, Inc.

for the

U.S. DEPARTMENT OF ENERGY

under Contract No. DE-AC05-84OR21400

DISTRIBUTION OF THIS DOCUMENT IS UNLIMITED 


\section{Contents}

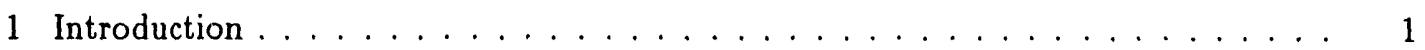

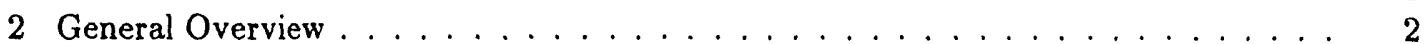

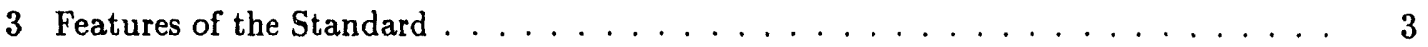

3.1 Basic Message Passing Routines . . . . . . . . . . . . . . . . . 3

3.1 .1 Receiving messages . . . . . . . . . . . . . . . . . . 4

3.1 .2 Sending messages . . . . . . . . . . . . . . . . . . 5

3.1.3 Other message passing utilities .................. 6

3.1.4 Buffering of messages by the system . . . . . . . . . . . . . 6

3.2 Process Groups . . . . . . . . . . . . . . . . . . . . 7

3.2.1 Creating and Managing Process Groups . . . . . . . . . . . . . . . 7

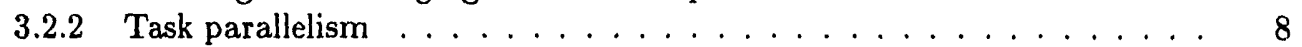

3.2.3 Examples of the use of subgroups . . . . . . . . . . . . . . . 9

3.3 Communication Contexts . . . . . . . . . . . . . . . . 11

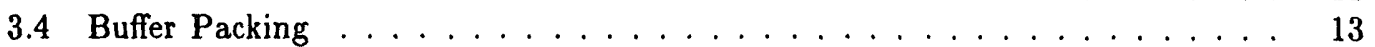

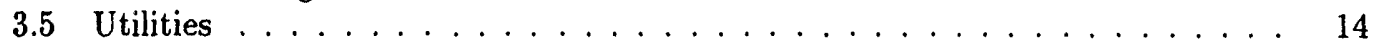

4 Discussion and Rationale . . . . . . . . . . . . . . . . . . . . 14

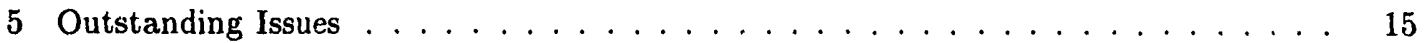

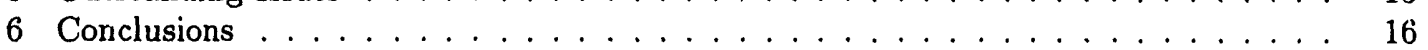

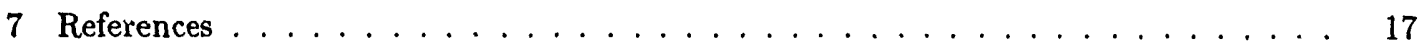




\title{
A PROPOSAL FOR A USER-LEVEL, MESSAGE PASSING INTERFACE IN A DISTRIBUTED MEMORY ENVIRONMENT
}

\author{
Jack J. Dongarra \\ Rolf Hempel \\ Anthony J. G. Hey \\ David W. Walker
}

\begin{abstract}
This paper describes Message Passing Interface 1 (MPI1), a proposed library interface standard for supporting point-to-point message passing. The intended standard will be provided with Fortran 77 and $\mathrm{C}$ interfaces, and will form the basis of a standard high level communication environment featuring collective communication and data distribution transformations. The standard proposed here provides blocking, nonblocking, and synchronized message passing between pairs of processes, with message selectivity by source process and message type. Provision is made for noncontiguous messages. Context control provides a convenient means of avoiding message selectivity conflicts between different phases of an application. The ability to form and manipulate process groups permits task parallelism to be exploited, and is a useful abstraction in controlling certain types of collective communication.
\end{abstract}




\section{Introduction}

This paper documents a proposal, initially made in November 1992, for a standard for performing point-to-point message passing between pairs of processes in a MIMD distributed memory computing system. Some modifications were made in January 1993, particularly in the approach to process groups, following input from a number of colleagues. An effort is currently underway to develop a more comprehensive standard for message-passing on distributed memory systems by July 1993. This effort involves a team of about 60 people made up of hardware and software vendors, and researchers from universities and government laboratories.

A small set of typed message passing routines form the core of the standard, and are augmented by support for features such as noncontiguous messages, communication contexts, and process groups. The proposed standard, called Message Passing Interface 1 (MPI1), includes only message passing between distinct pairs of processes, and thus does not address collective communication of any type, including broadcasts and reduction operations. We expect these types of communication will be included in the final version of the MPI standard. Other important standardization issues not addressed in detail include support for virtual communication channels, active messages, heterogeneous computing, performance tracing, and parallel 1/O. Thus, while MPI1 does not at this stage provide the flexibility and range of functionality that one would expect from a complete message passing environment, we regard it as forming the core of such an environment. In designing MPI1 we have tried to avoid imposing constraints that would hinder the future extensions necessary to address the issues mentioned above.

The main advantages of establishing a message passing standard are portability and easeof-use. In a distributed memory communication environment in which the higher level routines and/or abstractions are built upon lower level message passing routines the benefits of standardization are particularly apparent. Furthermore, the definition of a message passing standard, such as that proposed here, provides vendors with a clearly defined base set of routines that they can implement efficiently, or in some cases provide hardware support for, thereby enhancing scalability.

In designing MPI1 we have sought to make use of the most attractive features of a number of existing message passing systems, rather than selecting one of them and adopting it as the standard. Thus, MPI1 has been strongly influenced by work at the IBM T. J. Watson Research Center by Bala, Kipnis, Snir and colleagues [2,3], Intel's NX/2 [18], Express [17], nCUBE's Vertex [15], and PARMACS [11,13]. Other important contributions have come from Zipcode $[19,20]$, Chimp [6,7], PVM [8,21], and PICL [9].

One of the objectives of this paper is to promote a discussion within the concurrent computing research community of the issues that must be addressed in establishing a practical, portable, and flexible standard for message passing. This cooperative process began with a workshop on standards for message passing held in April 1992 [22], and continued with a second meeting in November 1992 when an organizational structure for developing a standard 
message passing interface was created. We believe the draft of MPI1 proposed here provides a good, concrete basis for continued discussion, and that it will contribute over the next few months to the development of an intermediate level message passing standard.

In Section 2 the rationale for an intermediate level standard is given. Section 3 presents the programming model assumed, and describes the main features of MPI1. Section 4 discusses the main decisions and compromises made in designing MPI1. Some important unresolved issues that must be addressed before MPIl can be regarded as complete are presented in Section 5. These include support for application topologies and heterogeneous computing, and a more general approach to process groups. Finally, Section 6 presents concluding remarks, and solicits the involvement of the research community in the development of a standard for a comprehensive message passing interface. Detailed specifications of the MPI1 routines are given in Appendix $\mathrm{A}$ in the form of manual pages.

\section{General Overview}

It is possible to consider defining a message passing standard at a number of levels. At the lowest level, closest to the hardware, might be syntactically simple routines for moving packets along wires. Above this channel-addressed level might be a process-addressed level (where there may be more than one process on each physical processor), such as that defined by NX or Vertex on the iPSC and nCUBE machines, the commercially-available Express communication environment, or the PARMACS message passing macros. Higher-level abstractions, for example, Linda [4,10], MetaMP [16], or Shared Objects [1,14], would lie above this level. Each level could be built using the level beneath, provided that the overhead in doing this was sufficiently low that the cumulative overhead incurred at the higher levels was small. These successive software levels form a series of layers, that with some stretch of the imagination resemble the multiple skins of an onion, with the hardware being at the center. We, therefore, call this the "Onion Skin Model" of the distributed communication environment. In deciding at which level to try to impose a standard it should be noted that different people might favor different types of standard. For example, a non-expert user would prefer to use high-level abstractions, such as virtual shared memory, so that details of the message passing are hidden. On the other hand, a compiler writer would like to produce a portable parallel compiler, and would like to use small, fast messages such as might be provided by a low-level standard. Finally, an expert application developer might be prepared to sacrifice some ease-of-use for additional speed, and so would prefer a intermediate level standard that provides a set of efficient primitives for point-to-point message passing. The standard proposed here is intended for use by such an application developer.

If the Onion Skin model is valid, then it makes sense to impose a standard that is also layered. However, the hardware of different distributed memory computing systems is sufficiently varied that it is difficult to impose a low-level standard that is efficient on all machines. 'iherefore, 
it is more appropriate to define a standard at an intermediate level, and to implement this as efficiently as possible on each machine. There is still the possibility of defining higher-level standards on top of this intermediate level. Thus, the intermediate-level standard will be open and extendable. It is the standardization of this intermediate level of point-to-point message passing between pairs of processes that is the focus of this paper.

\section{Features of the Standard}

Our programming model assumes some set of processes that communicate by point-to-point message passing. With each process is associated some memory directly accessible only by that process - there is no shared memory. In MPI1 it is assumed that processes are single threaded, though we expect the final MPI standard to permit multithreaded processes. Although the message passing paradigm is usually associated with distributed memory systems, it is not necessary to make any strong assumptions about the underlying hardware. The proposed message passing standard could also be implemented on shared memory machines and uniprocessor workstations. Note that the standard does not address the issue of how the processes are assigned to physical processing nodes. In general, this issue requires the development of machine-dependent static and dynamic load balancers, and lies outside the scope of the proposed standard.

MPI1 provides some support for task parallelism. To this end each process is assumed to be a member of one or more process groups, each of which is identified by a unique process Group ID number, or GID. The processes in a group can cooperate to perform tasks completely independently of other processes, and in this sense each group can behave like a distinct virtual machine. The concept of process groups is also important when designing collective comrnunication routines.

\subsection{Basic Message Passing Routines}

We now introduce the basic message passing routines that form the core of the proposed standard. These routines permit point-to-point message passing between pairs of processes, with message selectivity based explicitly on message type and source process, and implicitly on communication context. Communication contexts are explained in more detail in Section 3.3.

MPI1 provides three modes for sending and receiving messages: blocking, nonblocking, and synchronized. These different communication modes are explained below. The mode is passed as an argument to the send or receive routine. A nonblocking or blocking send routine may be matched by a nonblocking or blocking receive routine in any combination. However, a synchronized send must be matched by a synchronized receive.

Noncontiguous messages are handled by providing three variants of the send and receive routines. The first variant assumes contiguous messages, and MPI1 provides the routines MPI_CSEND and MPI_CRECV for such messages. The second deals with messages that are 
gathered from, or scattered to, a buffer with constant stride. This type of routine may be used when communicating rows of a distributed matrix that is stored by columns. The routines MPI_SSEND and MPI_SRECV are used in this case. The third variant deals with messages that are gathered from, or scattered to, a buffer in an arbitrary way. MPI1 provides the routines MPI_GSEND and MPI_GRECV for this purpose. This last case provides a mechanism for doing point-to-point scatter/gather operations between pairs of processes. The data blocks comprising the message may be of differing sizes and lie at arbitrary locations in the buffer gathered from or scattered to. The scatter/gather operations are controlled by a pair of arrays. The first of these arrays contains pointers into a buffer that indicates where the data for the message is coming from, or going to. The second array indicates how many data items are to be extracted from, or stored to, each location pointed to. For example, suppose in some spatially decomposed particle simulation we build a list of the particles that must be migrated to another process in each time step. This list is a set of indices into the data structure containing the particle information. The Fortran language requires that the scatter/gather locations be specified by an indirection vector that applies to a specific buffer. The $\mathrm{C}$ language permits pointer manipulation, so the memory location from which data are gathered, or to which data are scattered, can be more naturally expressed as an array of pointers. This is one of the few significant syntactic differences between the $\mathrm{C}$ and Fortran versions of MPI1. We expect the final version of the MPI standard will specify the scattering and gathering of data with an "iovec" data structure, as is used by the readv and writev routines of the Posix standard.

\subsubsection{Receiving messages}

The receipt of a message is said to be blocking if the receiving process suspends execution until all of the message has been received, i.e., until it has been placed in an application buffer on the receiving process. If a process attempts to perform a blocking receive that is not matched by a corresponding loosely synchronous send, execution will be suspended indefinitely on that process, resulting in full or partial deadlock.

A nonblocking receive takes place in two phases. First, a receive is posted on the receiving process, that is, the application provides a buffer that is to be used to store a specified incoming message. After this the receiving process can then continue to do useful work. However, at this stage receipt of the message is not guaranteed, and the data in the message should not yet be used by the receiving process. The nonblocking receive must be completed in a second phase that either calls the routine MPI_WAIT that blocks until the message is received, or periodically calls the routine MPI_STATS that checks on whether the message has been received into an application buffer. Between these periodic checks useful work can continue to be done by the receiving process, and once receipt is confirmed the message may be processed. Using the blocking mechanism (MPI_WAIT) to complete a nonblocking receive is usually done immediately before the message is to be used on the receiving process, thereby allowing the 
maximum potential for overlap of computation and communication. This approach is common when the amount of work that could be done between posting the receive and actually using the received data can be quantified at compile time. In more dynamic situations there may be an almost arbitrary amount of work that a process could do until an anticipated message arrives. In such cases it is common to periodically check for message receipt using MPI_STATS. At the application level, a blocking receive is conceptually the same as a nonblocking receive in which no useful work is done between the two phases, i.e., a call to an MPI1 receive routine in nonblocking mode immediately followed by a call to MPI_WAIT.

When a message is received in synchronized mode, the receiving process sends an acknowledgment to the sending process once the message has been completely received and placed in an application buffer. In the absence of hardware failures, and provided valid arguments are passed to the send and receive routines, message receipt is guaranteed.

\subsubsection{Sending messages}

The sending of a message is said to be blocking if the sending process suspends execution until all of the message has been sent, i.e., until the application buffer containing the message on the sending process is available for reuse. When this has occurred we say that "the message has cleared the buffer." It is not guaranteed that the message will actually be delivered to the destination process, and unless the application performs some additional handshaking, the sending process cannot know if the message was delivered.

A nonblocking send takes place in two phases. In the first phase the user calls an MPI1 send routine in nonblocking mode which initiates transmission of a specified message buffer to the destination process, and then returns. The sending process can then continue to do useful work, but during this time it is not guaranteed that the message has cleared the buffer, and it is a programming error to change it in any way. The nonblocking send must be completed in a second phase that either calls the routine MPI_WAIT that blocks until the message has been sent, or periodically calls the routine MPI_STATS that checks on whether the message has been sent or not. Between these periodic checks useful work can continue to be done by the sending process, and once the message has been sent the message buffer may then be safely modified. The routine MPI_STATS may be used to check for completion of a nonblocking send when there is an arbitrary amount of work that can be done between initiating and completing the send operation. A blocking send is conceptually the same as a nonblocking send in which no useful work is done between the two phases, i.e., a call to an MPI1 send routine in nonblocking mode immediately followed by a call to MPI_WAIT.

When a message is sent in synchronized mode, execution is suspended on the sending process until an acknowledgment has been received from the destination process indicating that message receipt has completed. For a message sent in synchronized mode the message is not buffered by the system, and upon delivery to the the destination process it is placed directly into the 
supplied application buffer.

\subsubsection{Other message passing utilities}

On systems that provide buffering for messages (see Section 3.1.4) it is sometimes necessary for a process to check whether it has any pending messages satisfying given selection criteria. MPI1 provides the routine MPI_PROBE for this purpose. A pending message is one that was sent in blocking or nonblocking mode, but for which a corresponding receive has not yet been posted on the destination process. Such messages may be buffered by the system on the destination process, thus MPI_PROBE queries the contents of the system message buffers. Note that MPI_PROBE differs from MPI_STATS which checks for delivery of a message into an application buffer.

Either, or both, of the type and source message selection criteria specified in an MPI1 receive routine, or the routine MPI_PROBE can have wildcard values. A wildcard value for the type or source indicates that this criterion is to be ignored in selecting messages on a destination process, so it is possible to select messages regardless of type and/or source. After it has been ascertained by a process that it has received a wildcarded message, or that it has such a message pending, the actual length, type, and/or source of the message can be determined by calling MPI_INFOL, MPI_INFOT, and MPI_INFOS, respectively.

The routine MPI_CANCEL can be used to cancel a specified nonblocking send or receive operation initiated previously. After returning from MPI_CANCEL the nonblocking operation is no longer active, and the status of the nonblocking operation is left indeterminate.

\subsubsection{Buffering of messages by the system}

In describing MPI1's message passing routines, we have tried to avoid making any unnecessary assumptions about the underlying communication mechanism. In this section we will touch on some implementation issues that affect application portability, and whether message delivery is guaranteed.

In general, a communication system has some buffering capacity, as would usually be the case if the underlying communication mechanism was asynchronous. In such cases, when a message sent in blocking or nonblocking mode arrives at a destination process it is placed directly in an application buffer if a corresponding receive has already been posted; otherwise, it is placed in a system buffer. Messages in a system buffer are referred to as "pending messages," and remain in a system buffer until a corresponding receive is posted, at which point they are moved to an application buffer, and effectively deleted from the system buffer. Since the system can only provide a finite amount of buffer space for pending messages, an asynchronous communication mechanism must deal with the possibility that an incoming message would cause a system buffer to overflow. A simple recourse in such a situation is to discard the message, and flag an error condition on the receiving process. It should be noted that this would not be detected as 
an error by the sending process.

MPI can also be implemented on top of a synchronous communication system with no buffering capacity. In this case there are no system buffers, so the possibility of one overflowing does not arise. On such systems, a message buffer remains volatile on the sending process until a corresponding receive is posted on the destination process, at which point the message is delivered. Since messages are not buffered, the routine MPI_PROBE always indicates that there no pending messages.

To write applications that are portable between machines with different underlying communication mechanisms, and between machines whose communication systems have differing (and usually unknown) buffering capacities, reliance on system buffering should be avoided [5].

Although a synchronous communication system can guarantee message delivery (in the absence of hardware failures and software bugs), it is more difficult for an asynchronous system to do so. Thus, requiring guaranteed message delivery as part of a message passing standard may not be appropriate.

\subsection{Process Groups}

\subsubsection{Creating and Managing Process Groups}

Process groups provide a means of handling task parallelism, as well as controlling which processes cooperate in collective communication tasks, such as broadcast and reduction operations. MPI1 does not include collective communication routines, however, the support provided for process groups in MPI1 is intended to be fully consistent with the use of process groups in collective communications, a standard for which we expect to be defined subsequently. Thus, within the context of MPI1 process groups are provided solely as a means of supporting task parallelism, in which different process groups work on different tasks.

A process group is identified by a unique process group ID, or GID, which is an integer greater than zero. When a parallel program starts up, the processes allocated to an application belong to the predefined group with GID $=$ ALL, where ALL is some integer assigned by the system. MPI1 provides two basic methods for creating a new group or groups. A new group can be created by each process in the group synchronously calling the routine MPI_DEFNG, which takes as its arguments the number of processes in the new group, and a list of the processes making up the group. A second routine, MPI_PARTG, is provided that allows a group to be partitioned into distinct subgroups based on the value of a specified key.

Information about group membership can be obtained using the routines MPI_GETID and MPI_INFOG. Given a process group with $n$ members, the processes in the group are uniquely labeled $0,1, \ldots, n-1$. These labels may be regarded as process ID numbers that are specific to a particular group, and will be referred to as Group Context Process ID numbers, or GCPIDs. A process has a different GCPID for each group of which it is a member. The routine MPI.GETID returns the GCPID of the calling process in a given group, or -1 if the process in not in the 
group. The routine MPI_INFOG can be used to determine which processes belong to a specified group of which the calling process is a member.

System memory is required to store information about the configuration of all currently defined groups. In order to make efficient use of this memory groups that are no longer needed by an application can be discarded, thereby freeing some memory for reuse. MPI provides the routine MPI_FREEG to discard a specified group. The routine MPI_FREEG must be called synchronously by all processes in the discarded group.

Finally, the routine MPI_SYNCG imposes a barrier synchronization on a specified group of processes.

All processes that are involved in an operation to produce, discard. or synchronize a group must perform the operation loosely synchronously, or full or partial deadlock may result.

\subsubsection{Task parallelism}

The routines discussed in Section 3.2.1 are concerned with creating, discarding, sychronizing, and inquiring about process groups. The use of groups to manage task parallelism will now be discussed. We consider three types of task parallelism, corresponding to the SIMD, SPMD, and MIMD programming models, each of which subsumes the former. In SIMD task parallelism each group of processes executes the same instructions on different data. For example, suppose we have two groups of processes of the same size, and want to find the fast Fourier transform (FFT) of two vectors of the same length. Then, one FFT can be done by one group and the other FFT by the second group, and processes in each group with the same GCPIDs will execute the same instructions. In SPMD task parallelism each process executes the same code, but different groups may execute different instructions. The groups are not required to be of the same size, but must be distinct. Finally, in MIMD task parallelism different executable programs are loaded into each group. It should be noted that MIMD task parallelism can be mimicked by SPMD task parallelism by having each group execute different branches of a conditional statement within a single executable program. As currently defined MPI1 supports SPMD task parallelism, but not MIMD task parallelism.

Two routines specifically for using groups to manage the SPMD style of task parallelism will now be introduced. MPI_PUSHG establishes an environment in which a specified group of processes is treated as if it were the only processes in use by the application, i.e., it establishes a process group context. MPI_POPG re-establishes the process group context in effect prior to the corresponding preceding call to MPI_PUSHG. The use of these routines is, perhaps, best demonstrated with an example. Suppose we have a piece of software that performs some task in parallel on $n$ processes, where $n$ is an input parameter passed to the software. In executing the parallel software, communication between the processes is based on the assumption that they are numbered $0,1, \ldots, n-1$. However, the actual PIDs of the processes in the group executing the software, in general, will not be labeled in this way since we are able to construct groups 


\begin{tabular}{ccc}
\hline PID & GID & GCPID \\
0 & 1 & 0 \\
1 & 1 & 1 \\
2 & 1 & 2 \\
3 & 1 & 3 \\
4 & 2 & 0 \\
5 & 2 & 1 \\
6 & 2 & 2 \\
7 & 2 & 3 \\
\hline
\end{tabular}

Table 1: Mapping of group context PIDs to PIDs

with arbitrary membership. However, the GCPIDs of the processes do run from 0 to $n-1$, so whenever the software refers to a source or destination process in the range 0 to $n-1$ this must be interpreted as a GCPID, which is then mapped to the corresponding PID. Thus, between a call to MPI_PUSHG and the corresponding subsequent call to MPI_POPG any reference to a process ID number is interpreted as a GCPID and is automatically mapped to the appropriate process ID number. For example, suppose the ALL group consists of 8 processes with process ID numbers $0,1, \ldots, 7$. Now suppose further that these processes have been partitioned so that the first four form one group with $G I D=1$, and the others form a second group with GID=2, and that the contexts for these groups have been established by calls to MPI_PUSHG. Then the GCPID associated with each process is as given in Table 1. Now, for example, if in the second group process 1 is required to send a message to process 3 , the process ID numbers are interpreted so the communication actually takes place between processes 5 and 7 . In this way a piece of software designed to execute on $n$ processes with PIDs 0 through $n-1$ will perform correctly within any group context.

After a call to MPI_PUSHG the predefined group ALL refers to the group whose context has just been established, and not to the original set of processes. The group can then be partitioned, and subgroups can be used to form new groups, by calling the routines MPI_PARTG and MPI_DEFNG. No reference may be made to any process or group outside the current group context. Group contexts may be nested.

A process must not be involved in any outstanding nonblocking communications within the current communication context (see Section 3.3) when calling MPI_PUSHG or MPI_POPG. All processes that are involved in an operation that changes the group context must perform the operation loosely synchronously, or full or partial deadlock may result.

\subsubsection{Examples of the use of subgroups}

To further clarify the use of subgroups in managing task parallelism we shall consider now some specific examples that use the MPI routines introduced in Sections 3.2.1 and 3.2.2. The first example is the solution of the shallow water equations on a sphere by the spectral transform method [23,25]. An important computational kernel of this application is the spectral transformation of a state variable defined on a rectangular longitude/latitude grid into a set of spectral 
ROWGRP = MPI_PARTG (ALI, MY_ROW)

COLGRP = MPI_PARTG (ALL, MY_COL)

Figure 1: Creation of row and column groups. Here MY_ROW and MY_COL are the position of a process in the logical $P \times Q$ process mesh.

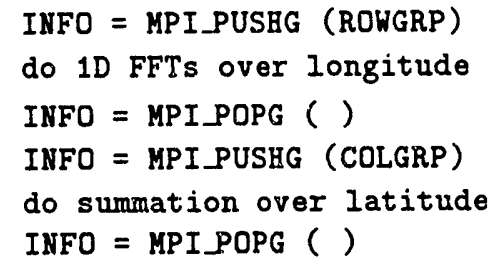

Figure 2: Pseudocode outline showing the use of process groups in the shallow water equatiun application.

coefficients. The spectral transform is evaluated in two phases. In the first phase a fast Fourier transform (FFT) is performed along each line of constant latitude in the grid. In the second phase the spectral transform is completed by taking a weighted integral over latitude of the Fourier coefficients. Numerically this is performed by weighted summation.

Suppose that the longitude/latitude grid is distributed in blocks over a two-dimensional, logical mesh of $P \times Q$ processes. Currently MPI1 does not provide a mechanism for establishing process topologies of this type, however, a proposal for extending MPIl to do this has been suggested by Hempel [12]. The processes in each row of the process mesh cooperate to evaluate the FFTs along a set of latitude lines. Then, the processes in each column cooperate to evaluate the spectral coefficients for a set of wavenumbers. The two phases of the spectral transform algorithm can be managed by partitioning the processes into row groups and column groups by making two calls to the routine MPI_PARTG, as shown in Figure 1.

The calls to MPI_PARTG are made once at the start of the application. Thereafter, the spectral transform of a state variable can be found by first establishing a process group context for the rows, and doing the FFTs over longitude for each latitude using a generic parallel FFT routine that assumes processes are numbered $0,1, \ldots, Q-1$. Then, a process group context for the columns is established, and the summation over latitude for each wavenumber is performed using a parallel routine that assumes processes are numbered $0,1, \ldots, P-1$. Thus, the pseudocode for the spectral transform algorithm is as as shown in Figure 2.

A second example of an application that might make use of process groups is an eventbased circuit simulation sode [24]. We are grateful to K. Yelick of the University of California, Berkeley, for suggesting this example. The circuit is decomposed into loosely coupled subcircuits with different computational loads. Each subcircuit is assigned to a process group, where the appropriate size of each group is determined by the computaticnal load associated with the 
subcircuit. Thus, the groups are of different sizes. The subcircuits communicate whenever there is a significant change in voltage, however, once the input voltages for a time step are known the linear system associated with each subcircuit can be solved independently using LU factorization. Suppose, for example, the circuit may be split into three subcircuits with computational loads for the $\mathrm{LU}$ factorization in the ratio 1:9:16. Then, we might assign one process to the first subcircuit, 9 processes to the second, and 16 processes to the third, as shown schematically in Figure 3. In the parallel LU solver each group of processes needs to be arranged as a two-dimensional mesh, so as in the shallow water equation example, it is necessary to be able to specify a topology for a group of processes. Given an initial set of processes, these can be divided into process groups by calling MPI.PARTG or MPI_DEFNG. Each process then establishes a group context prior to performing the $\mathrm{LU}$ solve phase in order to determine with which processes it must cooperate to solve the linear system for the subcircuit to which it is assigned. Each group calls the same parallel LU solve routine, which in its simplest form has as its arguments the coefficient matrix, the righthand side vector, the size of the matrix, and the number of processes, $P$ and $Q$, in each direction of the logical process mesh. Thus, the code would look something like the following,

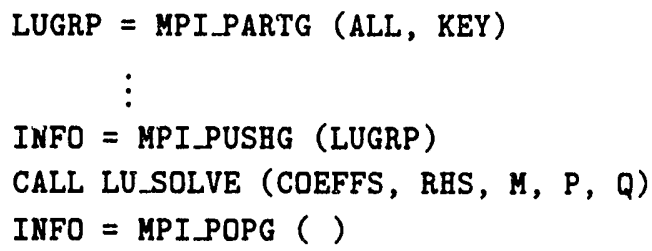

Note that the parallel LU solver may itself use row and column oriented subgroups. These would be set up within the parallel LU solve routine.

\subsection{Communication Contexts}

It is sometimes necessary to ensure that different streams of communication do not interfere with one another. For example, in an application with two distinct phases, each involving nonblocking communication, there is the possibility that one phase may intercept messages intended for the other phase. This situation can arise if the message selectivity criteria of the two phases overlap, as may be the case when using a "canned" concurrent software library in which the selectivity criteria, in general, are unknown. Communication contexts, first used in the Zipcode message passing system [19,20], provide a means of disambiguating such situations. In effect, a communication context provides a third selectivity criterion, in addition to type and source process, that may be used to control the receipt of messages. A communication context is uniquely labeled by a strictly positive integer called the Communication Context ID, or CCID. In MPI1 a communication context may be created by a call to MPI_NEWC, and a list of the current valid contexts may be obtained by calling MPI_INFOC. After invoking a pre- 


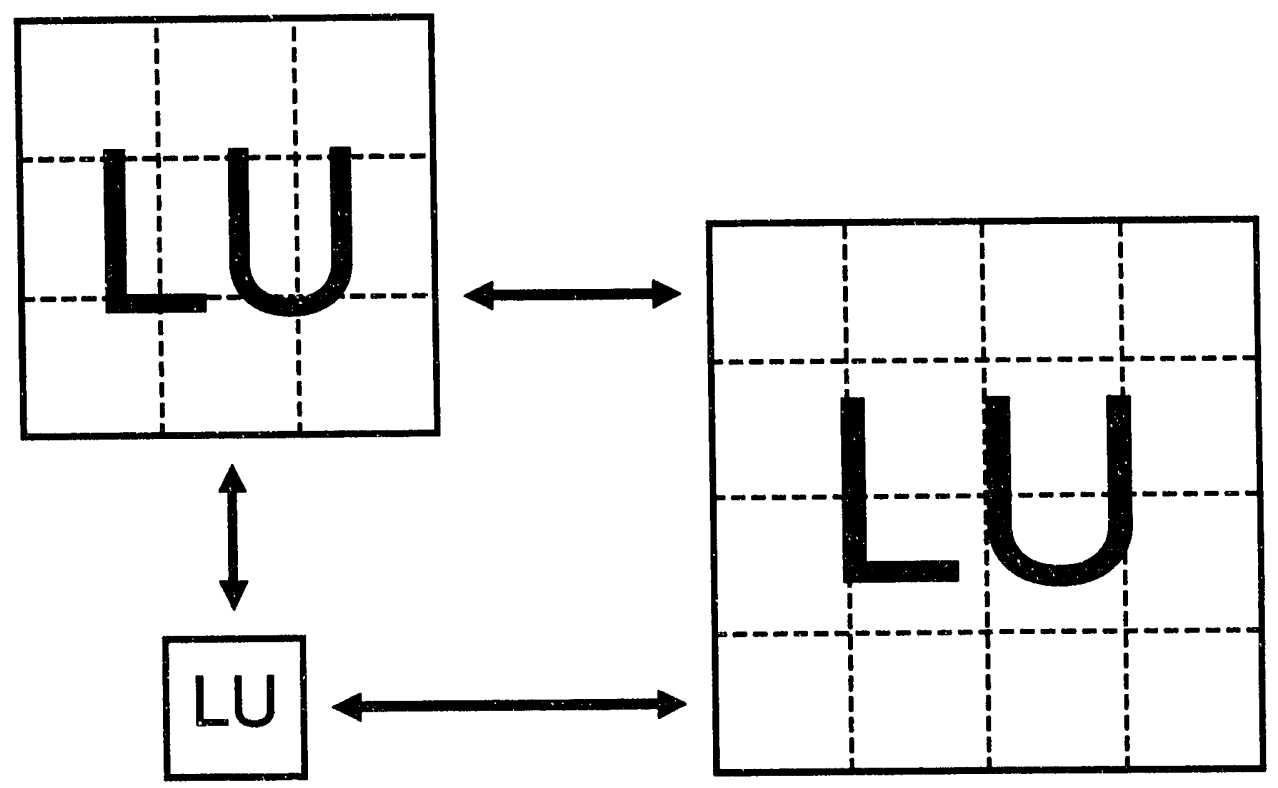

Figure 3: The division of processes into three groups of $1 \times 1,3 \times 3$, and $4 \times 4$ processes. Each group is assigned to a subcircuit, and independently performs a parallel LU solve. The arrows indicate the need for intermittent communication between the groups.

viously created communication context by calling MPI_PUSHC, all messages subsequently sent are tagged with that context, and only those messages so tagged may be received. The current communication context is terminated by a call to MPI_POPC, which restores the communication context in effect prior to the preceding call to MPI.PUSHC. Communication contexts may be nested.

As an example, suppose we want to evaluate $D=A B+C^{T}$, where $A, B, C$, and $D$ are all matrices. Then we might proceed as follows:

1. Initiate a nonblocking tran:pose of $C$

2. Call a concurrent library routine to find $A B$

3. Block until transpose of $C$ is complete

4. Add $C^{T}$ to $A B$ to form $D$

Here the task of transposing matrix $C$, which requires interprocess communication, is overlapped with the distinct task of evaluating the matrix product $A B$, which also requires communication. If the message selectivity criteria within the two tasks are not unique there is the possibility that one task will receive messages intended for the other task. Note that this example assumes a sophisticated communication processor that not only knows what messages need to be sent for the transpose, but also interleaves them with those of the matrix communication. Potential message conflicts can be avoided by establishing different contexts for the matrix multiplication and matrix transpose tasks. The MPI1 code fragment for this example would be as shown in Figure 4. 


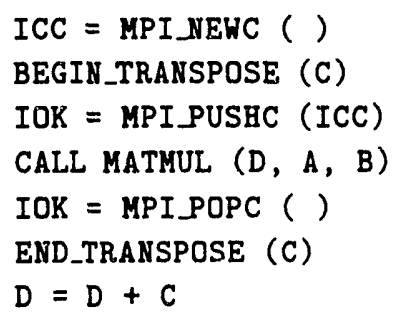

Figure 4: Code fragment illustrating the use of communication contexts

In the above example, the communication context ICC is first created by calling MPI_NEWC. The transposition of matrix $C$ is then initiated, with the communication context for this operation being the default context. Next, the routine MPI_PUSHC is called to establish the communication context with CCID number ICC. When MATMUL is then called only messages labeled with this communication context will be visible to the application, thereby, insulating the messages associated with the matrix multiplication from those of the matrix transposition. When MATMUL returns, the routine MPI_POPC is called to restore the default communication context. The routine END_TRANSPOSE blocks, if necessary, until the transposition is completed. If the communication associated with the transpose has already completed following the return from MPI_POPC, then END.TRANSPOSE just copies $C$ from a system to an application buffer.

Upon entering a program, or establishing a process group context by a call to MPI_PUSHG (see Section 3.2.2), a unique default communication context is established. A default communication context cannot be discarded, so a call to MPI_POPC when the current communication context is the default has no effect. When exiting a process group context by a call to MPI_POPG the communication context in effect prior to the preceding call to MPI_PUSHG is restored. Communication and process group contexts may be nested, but not misaligned.

\subsection{Buffer Packing}

As discussed in Section 3.1, point-to-point scatter/gather types of communication, in which data are gathered from a message buffer on the sending process, and subsequently scattered into a buffer on the receiving process, may be performed using different variants of the send and receive routines. Sometimes it may be necessary to gather/scatter data between multiple buffers that may be of differing data types. In the Fortran language this cannot be done by a single call to the MPI1 send/receive routines.

In this section we introduce routines that (1) gather data from a buffer and pack it contiguously into another buffer, and (2) scatter data into a buffer from a contiguous buffer. In all cases the buffers are on the same process, and no interprocess communication is required. These routines allow complex messages to be packed into a contiguous buffer on the sending process. 
This message can then be sent to the destination process using the routines MPI_CSEND and MPI_CRECV, where it can then be unpacked.

Two sets of pack/unpack routines are provided, and their syntax is very similar to that of the corresponding noncontiguous send/receive routines. The first pair of routines, MPI_SPACK and MPI_SUNPACK, handles the case in which data blocks of constant size are respectively gathered from, or scattered to, a buffer with constant stride. The second pair of routines, MPI_GPACK and MPI_GUNPACK, handles the case in which the data blocks may be of differing sizes and lie at arbitrary locations in the buffer gathered from, or scattered to.

\subsection{Utilities}

We expect the final version of MPI will include a set of routines for performing a variety of environmental management and inquiry functions. These routines, might for example, provide information on the machine the calling process is running on, the size of the system buffers available for interprocess communication, and other useful details. We also expect MPI to include routines for determining the date and time, and for finding the CPU time and elapsed wallclock time for a process. If a Posix standard exists for a routine then MPI should conform to it.

In general, the definition of these environmental and utility routines is deferred to later versions of MPI. The only utility routines provided in MPI1 are for error handling. Most of the routines in MPI1 return a value of -1 to indicate that an error has occurred. The nature and/or cause of the error can be determined by calling the routine MPI_ERROR. This returns an integer that indicates the error type applying to the most recent call to an MPIl routine. Among the types of error that would be indicated by a call to MPI_ERROR are the use of an invalid PID, GCPID, CCID, or MSGID; the loss of a message on a process due to a system buffer overflow; the use of an invalid block length or stride in one of the message packing routines; and so on. If the integer returned by MPI_ERROR is passed to the routine MPI_ETEXT, a string is returned giving a short description of the error which can then be output by the application. This way of handling errors is essentially the same as that used by PARMACS [13].

\section{Discussion and Rationale}

In this section we discuss the reasoning behind some of the decisions made in designing MPI1. In the design of this interface, one of the main concerns was to keep both the calling sequences simple and the range of options limited, while at the same time maintaining sufficient functionality. This clearly implies a compromise, and a good decision is vital if MPI1 is to be accepted as a useful standard.

In order to avoid potential programming errors, values of scalar variables are not returned through argument lists. In MPI1, routines are written as function calls rather than subroutine calls, which provides a mechanism for returning scalars. One consequence of this is that in order 
to determine the source, length, and/or type when a wildcarded message has been received in nonblocking mode, or is known to be pending following a call to MPLPROBE, it is necessary to call the information routines MPI_INF, MPI_INFOL, and/or MPI_INFOT.

It is not assumed in MPI1 that messages sent from one process to another are received in the order in which they were sent since some systems may use a non-deterministic routing scheme to avoid contention for communication links. Of course, even in such cases the correct order of messages could be recovered by the receiving process if each message was labeled by the sending process with a sequence number. Whether or not messages from one process to another arrive in the correct order has no impact on the definition of a standard, though clearly the assumption is vital to the correct functioning of many parallel algorithms.

MPI1 defines three modes for send and receiving messages, namely the blocking, nonblocking, and synchronized modes. We believe that these are the most widely used types of pointto-point communication operations, and in order to avoid too many varieties of send routine, some potentially useful functionality has been excluded from MPI1. For example, MPI1 does not include forced communication of the sort provided in Intel's NX/2 through the use of "force types." In forced communication, if a message sent in nonblocking mode is delivered to a process, and an application buffer has not already been made available for it by previously posting a receive, then the message is simply discarded, rather than being placed in a system buffer on the destination process. This functionality could be provided in MPI1 by reserving a certain range of types for forced communication, just as in $\mathrm{NX} / 2$. The justification for using forced communication is that it avoids some overhead, and thus is often faster. The main disadvantage is that it is the responsibility of the application to ensure that a receive is always posted prior to delivery of a forced message, otherwise the message will be lost.

In handling communication contexts MPI1 uses an approach that is independent of the message type selectivity mechanism. A different approach would be for each phase of an application to initially register the range of types it will use, and for a central message type registry to check for overlaps between the ranges claimed by different phases. An overlap would indicate to the application the potential for communication conflicts. The best approach is unclear. The first option would be more natural to the user, while for the second option communication context control functions would be easier to port onto most current parallel systems without major changes to the runtime systems. Thus, the question is how much MPI1 should be influenced by the presently available systems.

\section{Outstanding Issues}

In this section we outline a few of the issues that need to be addressed by MPI1, and some features that should be considered for inclusion in future versions of MPI1.

A number of extensions to the support provided by MPIl for process groups are possible. For example, currently in MPI1 the union of groups cannot be formed, nor is it possible for single 
processes to join or leave a group. Abstractions for permitting process groups to communicate with each other are another possible extension. MPI1 does not allow processes to be created or destroyed, or for different executable codes to be loaded into different processes, as would be required in order to support the MIMD style of task parallelism. The need for all these possible enhancements must be given careful consideration.

In MPI1 no explicit mechanism is provided to allow a process to inquire about the existence and membership of groups of which it is not a member. In a more general system it would be possible for a process to access information about any group. One way of doing this would be to have some processes dedicated to storing data about the current valid groups, and responding to requests for this information. Whenever, a group is created, discarded, or modified the processes involved must synchronize with one or more of the "group database" processes and inform them of the changes. Clearly, if there are too few such processes bottlenecks may develop in accessing their data; if there are too many then memory and compute power are wasted.

In the current version of MPIl a process group is formed by a collection of processes without any additional structure. Typical applications, on the other hand, have much more internal structure. For example, the solution of a partial differential equation on a $3 \mathrm{D}$ grid is usually performed by processes which are arranged in a corresponding structure. If the programming interface does not provide functions for defining that structure, the user must program the relationship of the logical position of a process and its identifier himself. Also, this information is not available for automatic tools which map neighboring processes onto neighboring hardware processors. Therefore, a mechanism such as that suggested by Hempel [12] for defining, and inquiring about, logical process topologies would be a useful addition to the message passing standard.

Another important consideration when extending MPI1 to handle heterogeneous distributed computing is the fact that different machines not only have different data formats, but also prefer different packet sizes. It would therefore appear that a table is needed that not only maps a PID number to an Internet address and process ID on the destination machine, but which also includes the target machine's preferred packet size.

\section{Conclusions}

We do not claim to provide the definitive answer to everyone's communication needs. Indeed, our insistence on simplicity precludes that. However, we believe the MPI1 routines proposed here will be useful as a basis for further discussion in the development of a standard for message passing in distributed memory environments. An MPI standards committee was formally instituted in November 1992, with the objective of providing a forum for discussion and of defining a standard message passing interface by July 1993. This committee is similar in structure and organization to that which developed the High Performance Fortran standard. Members of the distributed memory computing community who wish to become involved in the standardization 
process should send email to walker@msr.epm.ornl.gov by May 1, 1993.

\section{Acknowledgments}

This work was partially supported by the ESPRIT programme and the PPPE project. We gratefully acknowledge the participants of the First CRPC Workshop on Standards for Message Passing in a Distributed Memory Environment, and are grateful to the Center for Research on Parallel Computing for sponsoring this workshop. It is also a pleasure to acknowledge the helpful comments and suggestions of Vas Bala, Mark Debbage, Al Geist, William Gropp, Cherri Pancake, Paul Pierce, Peter Rigsbee, Anthony Skjellum, Marc Snir, and Joel Williamson.

\section{References}

[1] H. E. Bal. Programming Distributed Systems. Prentice Hall International, Hemel Hempstead, England, 1991.

[2] V. Bala and S. Kipnis. Process groups: a mechanism for the coordination of and communication among processes in the Venus collective communication library. Technical report, IBM T. J. Watson Research Center, October 1992. Preprint.

[3] V. Bala, S. Kipnis, L. Rudolph, and Marc Snir. Designing efficient, scalable, and portable collective communication libraries. Technical report, IBM T. J. Watson Research Center, October 1992. Preprint.

[4] N. Carriero and D. Gelernter. How to write parallel programs. ACM Computing Surveys, 21(3):323, September 1989.

[5] M. Debbage and M. Hill. Draft messaging ideas, revision 0.3. Technical report, Southampton University, UK, 1992. Preprint.

[6] Edinburgh Parallel Computing Centre, University of Edinburgh. CHIMP Concepts, June 1991.

[7] Edinburgh Parallel Computing Centre, University of Edinburgh. CHIMP Version 1.0 Interface, May 1992.

[8] G. Geist and V. Sunderam. Network based concurrent computing on the PVM system. Technical Report TM-11760, Oak Ridge National Laboratory, June 1991.

[9] G. A. Geist, M. T. Heath, B. W. Peyton, and P. H. Worley. A user's guide to PICL: a portable instrumented communication library. Technical Report TM-11616, Oak Ridge National Laboratory, October 1990.

[10] D. Gelernter. Generative communication in Linda. ACM Trans. Prog. Lang. Sys., 7(1):80$112,1985$. 
[11] R. Hempel. The ANL/GMD macros (PARMACS) in fortran for portable parallel programming using the message passing programming model - users' guide and reference manual. Technical report, GMD, Postfach 1316, D-5205 Sankt Augustin 1, Germany, November 1991.

[12] R. Hempel. A proposal for virtual topologies in MPI1. Technical report, GMD, Postfach 1316, D-5205 Sankt Augustin 1, Germany, November 1992.

[13] R. Hempel, H.-C. Hoppe, and A. Supalov. A proposal for a PARMACS library interface. Technical report, GMD, Postfach 1316, D-5205 Sankt Augustin 1, Germany, October 1992.

[14] D. Mallon, J. Nash, and P. Dew. Shared objects and its role in standardization. Technical report, Leeds University, UK, 1992. Preprint.

[15] nCUBE Corporation. nCUBE 2 Programmers Guide, r2.0, December 1990.

[16] S. Otto. MetaMP: a higher level abstraction for message passing programming. Technical report, Oregon Graduate Institute, Department of Computer Science, January 1991.

[17] Parasoft Corporation. Express Version 1.0: A Communication Environment for Parallel Computers, 1988.

[18] Paul Pierce. The NX/2 operating system. In Proceedings of the Third Conference on Hypercube Concurrent Computers and Applications, pages 384-390. ACM Press, 1988.

[19] A. Skjellum and A. Leung. Zipcode: a portable multicomputer communication library atop the reactive kernel. In D. W. Walker and Q. F. Stout, editors, Proceedings of the Fifth Distributed Memory Concurrent Computing Conference, pages 767-776. IEEE Press, 1990.

[20] A. Skjellum, S. Smith, C. Still, A. Leung, and M. Morari. The Zipcode message passing system. Technical report, Lawrence Livermore National Laboratory, September 1992.

[21] V. Sunderam. PVM: a framework for parallel distributed computing. Concurrency: Practice and Experience, 2(4):315-339, 1990.

[22] D. Walker. Standards for message passing in a distributed memory environment. Technical Report TM-12147, Oak Ridge National Laboratory, August 1992.

[23] D. W. Walker, P. H. Worley, and J. B. Drake. Parallelizing the spectral transform method - part II. Concurrency: Practice and Experience, 4:509-531, 1992.

[24] Chih-Po Wen. Timing simulation on a distributed memory multiprocessor. Master's thesis, University of California, Berkeley, CA, 1992.

[25] P. H. Worley and J. B. Drake. Parallelizing the spectral transform method - part I. Concurrency: Practice and Experience, 4:269-291, 1992. 


\section{Appendix A}

In this appendix we give Fortran specifications for the MPI1 routines. The $\mathrm{C}$ language specifications are not given explicitly, but are very similar, except for the routines dealing with arbitrary scatter/gather operations (MPI_GSEND, MPI_GRECV, MPI_GPACK and MPI_GUNPACK). In the synopses of the Fortran specifications of some of the routines, message buffers are referred to as integer arrays; however, real arrays can also be passed to these routines.

The appendix is consists of the following five sections.

1. Point-to-point message passing routines,

2. Support for process groups,

3. Support for buffer copying,

4. Support for communication contexts,

5. Utilities. 


\section{A.1 Point-to-Point Message Passing Routines}

In this section we provide specifications for the following point-to-point message passing and related routines.

- MPI_CANCEL Cancel nonblocking send or receive

- MPI_CRECV Receive contiguous message

- MPI_CSEND Send contiguous message

- MPI_GRECV Receive into buffer with arbitrary scatter

- MPI_GSEND Send from buffer with arbitrary gather

- MPI_INFOL Get length of pending or received message

- MPI_INFOS Get source of pending or received message

- MPI_INFOT Get type of pending or received message

- MPI_PROBE Check pending messages

- MPI_SRECV Receive into buffer with constant stride

- MPI_SSEND Send from buffer with constant stride

- MPI_STATS Check status of nonblocking send or receive

- MPI_WAIT Block until send or receive has completed

Message selectivity (within a communication context) is by source process and message type, either of which may have the "wildcard" value of -1 , indicating that any source and/or type is acceptable.

Nonblocking sends and receives return a message ID that is unique within the current group context. All other sends and receives return the number of bytes actually sent or received, or -1 if an error occurred. 
NAME

MPI_CANCEL Cancel a previously initiated nonblocking send or receive

SYNOPSIS

integer function MPI_CANCEL (msgid)

integer msgid

INPUT ARGUMENTS

msgid message identifier returned by a call to a nonblocking send or receive

\section{DESCRIPTION}

MPI_CANCEL cancels a previously issued nonblocking send or receive specified by the message identifier, msgid. Upon return the nonblocking send or receive is no longer active, and may or may not have completed.

\section{RETURN VALUE}

MPI_CANCEL returns 0 , or -1 if an error occurs. 
NAME

MPI_CRECV Receive a message contiguously into a buffer.

\section{SYNOPSIS}

integer function MPI_CRECV (mode, buf, source, type, maxlen)

integer mode

integer buf $(*)$

integer source

integer type

integer maxlen

\section{INPUT ARGUMENTS}

mode the mode of the receive (blocking, nonblocking, or synchronized)

source the ID number of the process sending the message

type the message type, or type mask

maxlen the maximum length of the message in bytes

\section{OUTPUT ARGUMENTS}

buf the application buffer into which the message is received.

\section{DESCRIPTION}

If mode has the system-defined value MPI_BLOCKING then the calling process blocks until a message of a specified type is received from a specified source into the application buffer buf. Deadlock will occur if no corresponding message is sent loosely synchronously by the source process.

If mode has the system-defined value MPI_NONBLOCKING then the calling process posts a receive for a message of a specified type from a specified source, and immediately returns. If mode has the system-defined value MPI_SYNCHRONIZED then the calling process blocks until the specified message has been received into the application buffer, buf, and then sends an acknowledgment to the source process before returning. The receive must be matched by a corresponding send, also done in synchronized mode.

For all modes, if source is -1 then selectivity by source is ignored. Similarly, if type is -1 then selectivity by type is ignored. Messages longer than maxlen bytes are truncated to maxlen bytes.

For all modes, the message received is stored contiguously in the buffer buf.

\section{RETURN VALUE}

Upon successful completion, if mode is MPI_BLOCKING or MPI_SYNCHRONIZED then MPI_CRECV returns the length of the message received in bytes. This will exceed maxlen bytes if the message was truncated. If mode is MPI_NONBLOCKING then MPI_CRECV returns the message ID number associated with the receive operation. 
A value of -1 is returned if an error occurs. 
NAME

MPI_CSEND Send a message contiguously from a buffer.

\section{SYNOPSIS}

integer function MPI_CSEND (mode, buf, dest, type, len)

integer mode

integer buf $(*)$

integer dest

integer type

integer len

\section{INPUT ARGUMENTS}

mode

buf

dest

type

len the mode of the send operation

the buffer containing the message to be sent

the ID number of the process to which the message is sent

the rnessage type

the length of the message in bytes

\section{DESCRIPTICN}

If mode has the system-defined value MPI_BLOCKING then MPI_CSEND sends a message of type type to process dest, and blocks until the message buffer, buf, is available for reuse.

if mode has the system-defined value MPI_NONBLOCKING then MPI_CSEND initiates transmission of a message of type type to process dest, and immediately returns. The message buffer, buf, should not be changed until the message is guaranteed to have been sent, i.e., to have "cleared ine buffer", by a call to MPI_WAIT, or by a call to MPI_STAT'S returning a nonnegative integer.

If mode has the system-defined value MPI_SYNCHRONIZED then MPI_CSEND sends a message of type type to process dest, and blocks until an acknowledgment is received from the destination proiess to indicate that message receipt has completed. The send must be matched by a corresponding receive, also cione in synchronized mode.

For all modes, the message consists of the len contiguous bytes in the buffer buf.

\section{RETUKN VALUE}

If mode is MPI_BLOCFING or MPI_SYNCHRONIZED then MPI_CSEND returns the number of bytes sent. If mode is MPI_NONBLOCKING then MPI_CSEND returns the message ID number associated with the send operation. A value of -1 is returned if an error occurs. 
NAME

MPI_GRECV Receive a message and scatter it arbitrarily into a buffer.

\section{SYNOPSIS}

integer function MPI_GRECV (mode, buf, source, type, nlist, ilist, nblks)

integer mode

integer buf $(*)$

integer source

integer type

integer $\operatorname{nlist}(*)$

integer ilist(*)

integer nblks

\section{INPUT ARGUMENTS}

mode the mode of the receive (blocking, nonblocking, or synchronized)

source the ID number of the process sending the message

type the message type, or type mask

nlist list of the number of bytes in each data block

ilist list of the location in buf at which each data block starts

nblks maximum number of data blocks to be scattered

\section{OUTPUT ARGUMENTS}

buf the application buffer into which the message is scattered

\section{DESCRIPTION}

If mode has the system-defined value MPI_BLOCKING then the calling process blocks until a message of a specified type is received from a specified source into the application buffer buf. Deadlock will occur if no corresponding message is sent loosely synchronously by the source process.

If mode has the system-defined value MPI_NONBLOCKING then the calling process posts a receive for a message of a specified type from a specified source, and immediately returns. If mode has the system-defined value MPI_SYNCHRONIZED then the calling process blocks until the specified message has been received into the application buffer, buf, and then sends an acknowledgment to the source process before returning. The receive must be matched by a corresponding send, also done in synchronized mode.

For all modes, if source is -1 then selectivity by source is ignored. Similarly, if type is -1 then selectivity by type is ignored. Messages longer than maxlen bytes are truncated to maxlen bytes.

For all modes, the way in which the data received are stored in the buffer buf is controlled by the arrays nlist and ilist. The data received are treated as a succession of data 
blocks, with the $i$ th block being of size nlist( $i$ ) bytes. This is stored in the buffer buf so that the start of the block is at ilist(i) bytes from the start of buf. The maximum number of data blocks received is nblks. It is assumed that all indices and numbering of data items begin at 0 . It is the responsibility of the user to ensure that buf is large enough to hold the data scattered into it.

\section{RETURN VALUE}

Upon successful completion, if mode is MPI_BLOCKING or MPI_SYNCHRONIZED then MPI_GRECV returns the total number of bytes received. If mode is MPI_NONBLOCKING then MPI_GRECV returns the message ID number associated with the receive operation. A value of -1 is returned if an error occurs. 
NAME

MPI_GSEND Send a message gathered arbitrarily from a buffer.

\section{SYNOPSIS}

integer function MPI_GSEND (mode, buf, dest, type, nlist, ilist, nblks)

integer mode

integer buf $(*)$

integer dest

integer type

integer $\operatorname{nlist}(*)$

integer ilist $(*)$

integer nblks

\section{INPUT ARGUMENTS}

$\begin{array}{ll}\text { mode } & \text { the mode of the send (blocking, nonblocking, or synchronized) } \\ \text { buf } & \text { the buffer containing the message to be sent } \\ \text { dest } & \text { the ID number of the process to which the message is sent } \\ \text { type } & \text { the message type } \\ \text { nlist } & \text { list of the number of bytes in each data block } \\ \text { ilist } & \text { list of the location in buf at which each data block starts } \\ \text { nblks } & \text { number of data blocks to be gathered }\end{array}$

\section{DESCRIPTION}

If mode has the system-defined value MPI_BLOCKING then MPI_GSEND sends a message of type type to process dest, and blocks until the message buffer, buf, is available for reuse.

If mode has the system-defined value MPI_NONBLOCKING then MPI_GSEND initiates transmission of a message of type type to process dest, and immediately returns. The message buffer, buf, should not be changed until the message is guaranteed to have been sent, i.e., to have "cleared the buffer", by a call to MPI_V: AIT, or by a call to MPI_STATS returning a nonnegative integer.

If mode has the system-defined value MPI_SYNCHRONIZED then MPI_GSEND sends a message of type type to process dest, and blocks until an acknowledgment is received from the destination process to indicate that message receipt has completed.

For all modes, the way in which the message sent is gathered from the buffer buf is controlled by the arrays nlist and ilist. The data are gathered in blocks, with the $i$ th block being of size nlist(i) bytes. This is gathered from the buffer buf starting at the location ilist ( $i$ ) bytes from the start of buf. The total number of data blocks gathered is nblks. It is assumed that all indices and numbering of data iterns begin at 0 . 


\section{RETURN VALUJE}

If mode is MPI_BLOCKING or MPI_SYNCHRONIZED then MPI_GSEND returns the number of bytes sent. If mode is MPI_NONBLOCKING then MPI_GSEND returns the message ID number associated with the send operation. A value of -1 is returned if an error occurs. 


\section{NAME}

MPIINFOL Determine the length of a pending or received message.

\section{SYNOPSIS}

integer function MPI_INFOL ()

\section{ARGUMENTS}

None

\section{DESCRIPTION}

MPI_INFOL determines the length in bytes of a pending or received message. It only returns a valid result if used directly after a call to a receive routine in blocking or synchronized mode, or directly after a call to MPI_STATS or MPI_PROBE that has returned a nonnegative integer.

\section{RETURN VALUE}

Directly after a call to a receive routine in blocking or synchronized mode, a call to MPI_WAIT, or a call to to MPI_STATS that returns a nonnegative integer, the routine MPI_INFOL returns the length in by'es of the message just received. If called directly after MPI_PROBE has returned a nonnegative number, MPI INFOL returns the length in bytes of the pending message. If there are no pending messages -1 is returned. 
NAME

MPI_INFOS Determine the source process of a pending or received message.

\section{SYNOPSIS}

integer function MPI_INFOS ()

\section{ARGUMENTS}

None

\section{DESCRIPTION}

MPI_INFOS determines the source process of a pending or received message. It only returns a valid result if used directly after a call to a receive routine in blocking or synchronized mode, or directly after a call to MPI_STATS or MPI_PROBE that has returned a nonnegative integer.

\section{RETURN VALUE}

Directly after a call to a receive routine in blocking or synchronized mode, a call to MPI_WAIT, or a call to to MPI_STATS that returns a nonnegative integer, the routine MPI_INFOS returns the ID number of the process that sent the message just received. If called directly after MPI_PROBE has returned a nonnegative number, MPI_INFOS returns the ID number of the process that sent the pending message. If there are no pending messages -1 is returned. 
NAME

MPI_INFOT Determine the type of a pending or received message.

\section{SYNOPSIS}

integer function MPI_INFOT ()

\section{ARGUMENTS}

None

\section{DESCRIPTION}

MPI_INFOT determines the type of a pending or received message. MPI_INFOT only returns a valid result if used directly after a call to a receive routine in blocking or synchronized mode, or MPI_WAIT, or directly after a call to MPI_PROBE or MPI_STATS that has returned a nonnegative integer.

\section{RETURN VALUE}

Directly after a call to a receive routine in blocking or synchronized mode, MPI..WAIT, or a call to MPI_STATS that returns a nonnegative integer, MPI_INFOT returns the type of the message just received. If called directly after MPI_PROBE has returned a nonnegative number, MPI_INFOT returns the type of the pending message. If there are no pending messages -1 is returned. 
NAME

MPI_PROBE Check for pending messages.

\section{SYNOPSIS}

integer function MPI_PROBE (source, type)

integer source

integer type

\section{INPUT ARGUMENTS}

source

the ID number of the process sending the message.

type

the message type, or type mask.

\section{DESCRIPTION}

MPI_PROBE checks if there is a message from a specified source and of a specified type awaiting receipt. That is, if there is a such a message stored in a system buffer for which a receive has not yet been posted. If source is -1 then this argument is ignored. Similarly, if type is - 1 .then this argument is ignored. Only messages sent using the routines sent in blocking or nonblocking mode may be buffered by the system on the receiving process, so it only makes sense to use MPI_PROBE to probe such messages.

\section{RETURN VALUE}

If a message satisfying the selectivity criteria is awaiting receipt MPI_PROBE returns the length of the message in bytes. Otherwise, -1 is returned. 
NAME

MPI_SRECV Receive a message and scatter it with constant stride into a buffer.

\section{SYNOPSIS}

integer function MPI_SRECV (mode, buf, source, type, lenblk, stride, nblks)

integer mode

integer buf $(*)$

integer source

integer type

integer lenblk

integer stride

integer nblks

\section{INPUT ARGUMENTS}

mode

the mode of the receive (blocking, nonblocking, or synchronized)

source

the ID number of the process sending the message

type

the message type, or type mask

lenblk

the size in bytes of each data block

stride

the number of bytes between the start of each data block

nblks

maximum number of data blocks to be scattered

\section{OUTPUT ARGUMENTS}

buf

the application buffer into which the message is scattered

\section{DESCRIPTION}

If mode has the system-defined value MPI_BLOCKING then the calling process k'ocks until a message of a specified type is received from a specified source into the application buffer buf. Deadlock will occur if no corresponding message is sent loosely synchronously by the source process.

If mode has the system-defined value MPI_NONBLOCKING then the calling process posts a receive for a message of a specified type from a specified source, and immediately returns. If mode has the system-defined value MPI_SYNCHRONIZED then the calling process blocks until the specified message has been received into the application buffer, buf, and then sends an acknowledgment to the source process before returning. The receive must be matched by a corresponding send, also done in synchronized mode.

For all modes, if source is -1 then selectivity by source is ignored. Similarly, if type is -1 then selectivity by type is ignored. Messages longer than maxlen bytes are truncated to maxlen bytes.

For all modes, the data received are treated as a succession of data blocks, each of length lenblk bytes. Data blocks are placed in the buffer buf so that the start of successive 
blocks are separated by stride bytes. The maximum number of data blocks received is nblks. It is the responsibility of the user to ensure that buf is large enough to hold the data scattered into it.

\section{RETURN VALUE}

Upon successful completion, if mode is MPI_BLOCKING or MPI_SYNCHRONIZED then MPI_SRECV returns the length of the message received in bytes. If mode is MPI_NONBLOCKING then MPI_SRECV returns the message ID number associated with the receive operation. A value of -1 is returned if an error occurs. 
NAME

MPI_SSEND Send a message gathered with constant stride from a buffer.

\section{SYNOPSIS}

integer function MPI_SSEND (mode, buf, dest, type, lenblk, stride, nblks)

integer mode

integer buf $(*)$

integer dest

integer type

integer lenblk

integer stride

integer nblks

\section{INPUT ARGUMENTS}

mode

buf

dest

type

lenblk

stride

nblks the mode of the send (blocking, nonblocking, or synchronized) the buffer containing the message to be sent the ID number of the process to which the message is sent the message type the size in bytes of each data block the number of bytes between the start of each data block number of data blocks to be gathered

\section{DESCRIPTION}

If mode has the system-defined value MPI_BLOCKING then MPI_SSEND sends a message of type type to process dest, and blocks until the message buffer, buf, is available for reuse.

If mode has the system-defined value MPI_NONBLOCKING then MPI_SSEND initiates transmission of a message of type type to process dest, and immediately returns. The message buffer, buf, should not be changed until the message is guaranteed to have been sent, i.e., to have "cleared the buffer", by a call to MPI_WAIT, or by a call to MPI_STATS returning a nonnegative integer.

If mode has the system-defined value MPI_SYNCHRONIZED then MPI_SSEND sends a message of type type to process dest, and blocks until an acknowledgment is received from the destination process to indicate that message receipt has completed.

For all modes, the data sent are gathered from the buffer buf in blocks, each of length lenblk bytes. The start of successive data blocks are separated by stride bytes in the buffer buf. The total number of data blocks gathered is nblks.

\section{RETURN VALUE}

If rode is MPI_BLOCKING or MPI_SYNCHRONIZED then MPI_SSEND returns the 
number of bytes sent. If mode is MPI_NONBLOCKING then MPI_SSEND returns the message ID number associated with the send operation. A value of -1 is returned if an error occurs. 
NAME

MPI_STATS Check the status of a nonblocking send or receive operation.

\section{SYNOPSIS}

integer function MPI_STATS (msgid)

integer msgid

\section{INPUT ARGUMENTS}

msgid

message identifier returned by a call to a nonblocking send or receive

\section{DESCRIPTION}

If the message identifier, msgid, refers to a message being sent in nonblocking mode, then MPI_STATS checks if the message has cleared the message buffer yet. If it has, then the message buffer is available for reuse. If the message identifier, msgid, refers to a message being received in nonblocking mode, then MPI_STATS checks if message receipt has been completed yet, i.e., if the incoming message has been placed in an application buffer. If it has, then the data received into the buffer is available for use.

\section{RETURN VALUE}

MPI_STATS returns the number of bytes sent or received if the nonblocking send or receive operation has completed. Otherwise, -1 is returned. 
NAME

MPI_WAIT

Block until a nonblocking send or receive operation has completed.

\section{SYNOPSIS}

integer function MPI_WAIT (msgid)

integer msgid

\section{INPUT ARGUMENTS}

msgid

message identifier returned by a call to a nonblocking send or receive

\section{DESCRIPTION}

If the message identifier, msgid, refers to a message being sent in nonblocking mode, then MPI_WAIT blocks until the message has cleared the message buffer. Upon return from such a call to MPI_WAIT the message buffer is available for reuse, but receipt of the message by the destination process is not guaranteed. If the message identiner, ms $\mathrm{g}-\mathrm{A}$. refers to a message being received in nonblocking mode, then MPI_WAIT blocks until message receipt has been completed. The data received into the message buffer is then available for use.

\section{RETURN VALUE}

On successful completion MPI_WAIT returns the number of bytes sent or received. Other wise, -1 is returned. 


\section{A.2 Support for Process Groups}

In this scction specifications for the following routines for supporting process groups are given.

- MPI_DEFNG Create a group from a list of processes

- MPI_FREEG Discard a group

- MPI_GETID Determine GCPID of calling process in a group

- MPI_INFOG Determine processes in a group

- MPI_PARTG Partition a group

- MPI_POPG Restore previous group context

- MPI_PUSHG Establish new group context

- MPI_SYNCG Synchronize a group of processes 
NAME

MPI_DEFNG Define a group of processes.

\section{SYNOPSIS}

integer function MPI_DEFNG (nprocs, plist)

integer nprocs

integer plist $(*)$

INPUT ARGUMENTS

nprocs the number of processes in the new group

plist a list of nprocs process ID numbers

\section{DESCRIPTION}

MPI_DEFNG creates a new group consisting of the nprocs processes whose ID numbers are listed in the array plist. The new group can subsequently be partitioned by calls to MPI_PARTG. MPI_DEFNG must be called synchronously by all the processes listed in plist.

\section{RETURN VALUE}

On successful completion MPI_DEFNG returns the unique group ID number of the newly formed group. If an error occurs a value of -1 is returned. 


\section{NAME}

MPI_FREEG Discard a specified group.

\section{SYNOPSIS}

integer function MPI.FREEG (gid)

integer gid

\section{INPUT ARGUMENTS}

gid

the group ID number of the group to be discarded

\section{DESCRIPTION}

MPI_FREEG may be used to free memory that stores information about groups that are no longer needed. The group gid is discarded, and may not be referred to subsequently. MPI_FREEG must be called synchronously by all processes in the group gid.

\section{RETURN VALUE}

On successful completion MPI_FREEG returns 0 . Otherwise -1 is returned. 
NAME

MPI_GETID Determine the group context PID of the calling process for a specified group ID number.

\section{SYNOPSIS}

integer function MPI_GETID (gid)

integer gid

\section{INPUT ARGUMENTS}

gid

the group ID for which the group context. PID is required

\section{DESCRIPTION}

MPI_GETID determines the group context PID of the calling process within the group gid. A value of -1 is returned if the calling process is not in the group gid.

\section{RETURN VALUE}

MPI_GETID returns the group context PID of the calling process within the group gid.

A value of -1 is returned if the calling process is not in the group gid. 
NAME

MPI_INFOG

Determine the number of processes in a group, and return a list of the PID numbers of the group members.

\section{SYNOPSIS}

integer function MPI_INFOG (gid, maxlis, plist)

integer gid

integer maxlis

integer plist $(*)$

\section{INPUT ARGUMENTS}

gid

a group ID number

maxlis

the maximum size of the array plist

\section{OUTPUT ARGUMENTS}

plist

a list of the PID numbers of the processes in group gid

\section{DESCRIPTION}

MPI_INFOG determines the number of processes the group gid, and returns a list of the PID numbers of the group members in the array plist. The calling process must be a member of the group gid. If there are more than maxlis processes in group gid, only the PID numbers of maxlis of them are returned in plist.

\section{RETURN VALUE}

On successful completion MPI_INFOG returns the number of processes in the group gid, or -1 if an error occurs. 
NAME

MPI_PARTG Partition a group into subgroups.

\section{SYNOPSIS}

integer function MPI_PARTG (gid, key)

integer gid

integer key

INPUT ARGUMENTS

gid the ID number of the group to be partitioned

key the key whose value determines the partitioning

\section{DESCRIPTION}

MPI_PARTG partitions the group gid into subgroups according to the value of key. All processes for which key has the same value form a distinct subgroup. MPI_PARTG must be called synchronously by all processes in the group gid.

\section{RETURN VALUE}

On successful completion MPI_PARTG returns the unique GID number of the subgroup to which the calling process belongs. Otherwise, -1 is returned. 
NAME

MPI_POPG Re-establish former process group context.

\section{SYNOPSIS}

integer function MPI_POPG ( )

\section{ARGUMENTS}

None

\section{DESCRIPTION}

MPI_POPG re establishes the process group context that was in effect before the preceding call to MPI_PUSHG. MPI_POPG must be called synchronously by all processes in the group whose context was established by the preceding call to MPI_PUSHG. The calling process must not be involved in any nonblocking communication within the current communication context when calling MPI_POPG.

\section{RETURN VALUE}

On successful completion MPI_POPG returns the process group ID number of the group whose context is re-established. Otherwise, -1 is returned. 
NAME

MPI_PUSHG Establish a new group context.

\section{SYNOPSIS}

integer function MPI_PUSHG (gid)

integer gid

\section{INPUT ARGUMENTS}

gid the group ID number of the context to be established

\section{DESCRIPTION}

A call to MPI_PUSHG establishes an environment in which it appears to the processes in the group gid that they are the only processes in use by the application. This environment is called the process group context of gid. The effect of a call to MPI_PUSHG is nullified by the next subsequent call to MPI_POPG, which re-establishes the process group context that was in effect before the call to MPI_PUSHG. If the group gid contains $n$ processes, then within the group context of gid each process is labeled by a unique integer between 0 and $n-1$, referred to as its group context PID. Processes may only be referenced by their group context PIDs, which are automatically mapped to the corresponding process ID numbers by the system. It is an error to refer to any process ID number outside the range 0 to $n-1$, and the processes in group gid may not communicate with processes outside the group. Groups created outside the current group context by calls to MPI_DEFNG, or MPI_PARTG may not be referenced. Groups created within the current group context may not be referenced after exiting the context by calling MPI_POPG. Within a group context the group ALL refers to just the processes in the current group context. Group contexts may be nested. MPI_PUSHG must be called synchronously by all processes in the group gid. The calling process must not be involved in any nonblocking communication within the current communication context when calling MPI_PUSHG.

\section{RETURN VALUE}

On successful completion MPI_PUSHG returns the number of processes in the group gid. Otherwise -1 is returned. 


\section{NAME}

MPI_SYNCG Synchronize processes.

\section{SYNOPSIS}

integer function MPI_SYNCG (gid)

integer gid

\section{ARGUMENTS}
gid
a process group ID

\section{DESCRIPTION}

MPI_SYNCG performs a barrier synchronization involving all processes in the group gid, of which the calling process must be a member.

\section{RETURN VALUE}

On successful completion MPI_SYNCG returns 0 . Otherwise, -1 is returned. 


\section{A.3 Support for Buffer Copying}

In this section specifications for the following routines for packing data into and out of message buffers are given.

- MPI_SPACK Gather data with constant stride

- MPI_SUNPACK Scatter data with constant stride

- MPI_GPACK General-purpose gather routine

- MPI_GUNPACK General-purpose scatter routine 
NAME

MPI_SPACK Pack data blocks into a buffer with constant stride.

SYNOPSIS

integer function MPI_SPACK (buf, lenblk, stride, nblk, msg)

integer buf $(*)$

integer lenblk

integer stride

integer nblk

integer $\mathrm{msg}(*)$

\section{INPUT ARGUMENTS}

buf

buffer from which data are to be gathered

lenblk size of each data block in bytes

stride number of bytes between successive blocks in buffer buf

nblk number of data blocks to be gathered

OUTPUT ARGUMENTS

msg

buffer in which the gathered data is packed

\section{DESCRIPTION}

MPI_SPACK gathers data from the buffer buf and packs it contiguously into the buffer msg. In buf the data blocks consist of lenblk bytes, with the starts of successive blocks being separated by a constant stride bytes. The number of blocks gathered in nblk. The most common use of MPI_SPACK is to fill a message buffer for subsequent communication.

\section{RETURN VALUE}

Upon successful completion MPI_SPACK returns the total length of the message in bytes. Otherwise, -1 is returned. 
NAME

MPI_SUNPACK Unpack data blocks from a buffer with constant stride.

\section{SYNOPSIS}

integer function MPI_SUNPACK (buf, lenblk, stride, nblk, msg)

integer buf $(*)$

integer lenblk

integer stride

integer nblk

integer $\mathrm{msg}(*)$

\section{INPUT ARGUMENTS}

lenblk

stride

nblk

msg size of each data block in bytes number of bytes between successive blocks in buffer buf number of data blocks to be scattered

buffer in which the data to be scattered are packed

\section{OUTPUT ARGUMENTS}

buf buffer to which data are to be scattered

\section{DESCRIPTION}

MPI_SUNPACK unpacks contiguous data from the buffer msg and scatters it with constant stride into the buffer buf. Successive contiguous blocks of lenblk bytes are extracted from msg and copied to buf so that the first such block is aligned with the start of buf, and the start of successive blocks is separated by stride bytes. A total of nblk data blocks are unpacked. The most common use of MPI_SUNPACK is to unpack data received from another process. It is the responsibility of the user to ensure that buf is large enough to hold the data unpacked into it.

\section{RETURN VALUE}

Upon successful completion MPI_SUNPACK returns the total length of the message in bytes. Otherwise, -1 is returned. 
NAME

MPI_GPACK General routine for packing data blocks into a buffer.

\section{SYNOPSIS}

integer function MPI_GPACK (buf, nlist, ilist, nblk, msg)

integer buf( $(*)$

integer nlist $(*)$

integer ilist $(*)$

integer nblk

integer $\mathrm{msg}(*)$

\section{INPUT ARGUMENTS}

buf buffer from which data are to be gathered

nlist list of the number of bytes in each block

ilist list of the location in buf at which each data block starts

nblk number of data blocks to be gathered

\section{OUTPUT ARGUMENTS}

msg buffer into which the gathered data are packed

\section{DESCRIPTION}

MPI_GPACK extracts nblk data blocks from the buffer buf and packs them contiguously into the buffer msg according to the information in the arrays nlist and ilist. The $i$ th data block extracted consists of the contiguous nlist( $i$ ) bytes starting at the location ilist ( $i$ ) bytes from the start of buf. It is assumed that all indices and numbering of data items begin at 0 . The most common use of MPI_GPACK is to fill a message buffer for subsequent communication.

\section{RETURN VALUE}

Upon successful completion MPI_GPACK returns the total length of the message in bytes. Otherwise, -1 is returned. 
NAME

MPI_GUNPACK General routine for unpacking data blocks from a buffer.

\section{SYNOPSIS}

integer function MPI_GUNPACK (buf, nlist, ilist, nblk, msg)

integer buf $(*)$

integer nlist $(*)$

integer ilist $(*)$

integer nblk

integer $\mathrm{msg}(*)$

\section{INPUT ARGUMENTS}

msg buffer from which the data to be scattered are unpacked

nlist list of the number of bytes in each block

ilist list of the location in buf at which each data block starts

nblk number of data blocks to be scattered

\section{OUTPUT ARGUMENTS}

buf

buffer into which data are to be scattered

\section{DESCRIPTION}

MPI_GUNPACK takes nblk successive contiguous data blocks from the buffer msg and unpacks them into the buffer buf according to the information in the arrays nlist and ilist. The $i$ th data block unpacked consists of nlist(i) contiguous bytes, and is copied to the buf so that the start of the block is aligned with the location ilist(i) bytes from the start of buf. It is assumed that all indices and numbering of data items begin at 0. The mosi common use of MPI_GUNPACK is to unpack a message received from another process. It is the responsibility of the user to ensure that buf is large enough to hold the data unpacked into it.

\section{RETURN VALUE}

Upon successful completion MPI_GUNPACK returns the total length of the message in bytes. Otherwise, -1 is returned. 


\section{A.4 Support for Communication Contexts}

In this section specifications for the following routines for managing communication contexts are given,

- MPI_INFOC Get information on valid communication contexts

- MPI_NEWC Create a new communication context

- MPI_POPC Restore a communication context

- MPI_PUSHC Establish a new communication context 


\section{NAME}

MPI_INFOC Get information about valid communication contexts

\section{SYNOPSIS}

integer function MPI_INFOC (maxlis, ilist)

integer maxlis

integer ilist $(*)$

\section{INPUT ARGUMENTS}

maxlis maximum number of communication context ID numbers in the array ilist

\section{OUTPUT ARGUMENTS}

ilist a list of communication context ID numbers

\section{DESCRIPTION}

MPI_INFOC determines the number of communication contexts that have been created for the current process group context, and returns a list of the corresponding communication context ID numbers in the array ilist. The first entry in ilist is always the ID number of the default communication context. If the number of ID numbers exceeds maxlis, then only maxlis are returned in the array ilist.

\section{RETURN VALUE}

On successful completion MPI_INFOC returns the number of communication contexts. Otherwise, -1 is returned. 
NAME

MPI_NEWC Create a new communication context.

\section{SYNOPSIS}

integer function MPI_NEWC ( )

\section{ARGUMENTS}

None

\section{DESCRIPTION}

MPI_NEWC creates a new communication context within the scope of the current process group context.

\section{RETURN VALUE}

On successful completion MPI_NEWC returns the unique ID number of the new communication context. Otherwise -1 is returned. 
NAME

MPI_POPC Re-establish former communication context.

\section{SYNOPSIS}

integer function MPI.POPC ( )

\section{ARGUMENTS}

None

\section{DESCRIPTION}

MPI_POPC re-establishes the communication context that was in effect before the preceding call to MPI_PUSHC.

\section{RETURN VALUE}

On successful completion MPI_POPC returns the ID number of the communication context that is re-established. Otherwise, -1 is returned. 


\section{NAME}

MPI_PUSHC Establish a new communication context.

\section{SYNOPSIS}

integer function MPI_PUSHC (ccid)

integer ccid

\section{INPUT ARGUMENTS}

ccid

the ID number of the communication context to be established

\section{DESCRIPTION}

MPI_PUSHC sets the current communication context to that given by the communication context ID number, ccid. This communication context stays in effect until the subsequent corresponding call to MPI_POPC, or until the next call to MPI_POPG, which destroys all the communication contexts of the process group context being exited. MPI_PUSHC must be called by all processes in the current process group context.

\section{RETURN VALUE}

On successful completion MPI_PUSHC returns 0. Otherwise -1 is returned. 


\section{A.5 Utilities}

In this section specifications for the following utility routines are given,

- MPI_ERROR Determine the current MPI error status

- MPI_ETEXT Get text string corresponding to error status 
NAME

MPI_ERROR Determine error status following a call to MPII

\section{SYNOPSIS}

integer function MPI_ERROR()

\section{ARGUMENTS}

None

\section{DESCRIPTION}

MPI_ERROR returns an integer giving the error status of the preceding call to an MPI1 routine.

\section{RETURN VALUE}

The meaning of the error status returned by MPI_ERROR is given in the table below. Additional entries may be added later.

\begin{tabular}{|c|l|}
\hline Error status & Meaning \\
0 & No error \\
1 & Invalid PID used \\
2 & Invalid GID used \\
3 & Invalid MSGID used \\
4 & Invalid CCID used \\
5 & Invalid GCPID used \\
6 & Invalid message buffer size \\
7 & Invalid stride in MPI_SPACK/MPI_SUNPACK \\
8 & Invalid block size in pack/unpack routine \\
9 & Invalid data item size in pack/unpack routine \\
10 & System buffer overflow \\
11 & Too many communication contexts \\
12 & Too many group contexts \\
\hline
\end{tabular}




$$
-60-
$$

NAME

MPI_ETEXT Give string describing the error status

\section{SYNOPSIS}

character*80 function MPI_ETEXT (ierrno)

integer ierrno

\section{INPUT ARGUMENTS}

ierrno

The error status

\section{DESCRIPTION}

MPI_ETEXT gives a brief description of the error corresponding to the value of the error status integer ierrno.

\section{RETURN VALUE}

MPI_ETEXT returns a string describing the error status. 
ORNL/TM-12231

\section{INTERNAL DISTRIBUTION}

1. B. R. Appleton

2. J. Choi

3-4. T. S. Darland

5. E. F. D'Azevedo

6-10. J. J. Dongarra

11. G. A. Geist

12. L. J. Gray

13. M. R. Leuze

14. E. G. $\mathrm{Ng}$

15. C. E. Oliver

16. B. W. Peyton

17-21. S. A. Raby

22. C. H. Romine
23. T. H. Rowan

24-28. R. F. Sincovec

29-33. D. W. Walker

34-38. R. C. Ward

39. P. H. Worley

40. Central Research Library

41. ORNL Patent Office

42. K-25 Applied Technology Library

43. Y-12 Technical Library

44. Laboratory Records - RC

45-46. Laboratory Records Department

\section{EXTERNAL DISTRIBUTION}

47. Giovanni Aloisio, Dipt. di Elettrotecnica ed Elettronica, Universita di Bari, Via Re David 200, 70125 Bari, Italy

48. Ed Anderson, Mathematical Software Group, Cray Research Incorporated, 655F Lone Oak Drive, Eagan, MN 55121

49. Ian G. Angus, Boeing Computer Services, M/S 7L-22, P. O. Box 24346, Seattle, WA 98124-0346

50. Marco Annaratone, Digital Equipment Corporation, 146 Main Street MLO15/U46, Maynard, MA 01754

51. Vasanth Bala, IBM T. J. Watson Research Center, P. O. Box 218, Yorktown Heights, NY 10598

52. Donald M. Austin, 6196 EECS Bldg ., University of Minnesota, 200 Union Street, S.E., Minneapolis, MN 55455

53. Edward H. Barsis, Computer Science and Mathematics, P. O. Box 5800, Sandia National Laboratories, Albuquerque, NM 87185

54. Eric Barton, Meiko Limited, 650 Aztec West, Bristol BS12 4SD, United Kingdom

55. Adam Beguelin, Carnegie Mellon University, School of Computer Science, 5000 Forbes Avenue, Pittsburgh, PA 15213-3890

56. Siegfried Benker, Institute for Statistics and Computer Science, University of Vienna, A-1210 Vienna, Austria

57. Ed Benson, Digital Equipment Corp., 146 Main Street, ML01-5/U46, Maynard, MA 01754 
58. Roger Berry, NCUBE Corporation, 4313 Prince Road, Rockville, MD 20853

59. Scott Berryman, Yale University, Computer Science Department, 51 Prospect Street, New Haven, CT 06520

60. Robert Bjornson, Department of Computer Science, Box 2158 Yale Station, New Haven, CT 06520

61. Peter Brezany, Institute for Statistics and Computer Science, University of Vienua, A-1210 Vienna, Austria

62. Roger W. Brockett, Wang Professor of EE and CS, Division of Applied Sciences. Harvard University, Cambridge, MA 02138

63. Clemens H. Cap, University of Zurich, Department of Computer Science, Winterthurerstr. 190, CH-8057 Zurich, Switzerland

64. John Cavallini, Acting Director, Scientific Computing Staff, Applied Mathematical Sciences, Office of Energy Research, U. S. Department of Energy, Washington, DC 20585

65. Siddhartha Chatterjee, RIACS, Mail Stop T045-1, NASA Ames Research Center, Moffett Field, CA 94035-1000

66. Kuo-Ning Chiang, MacNeil-Schwendler Corporation, 815 Colorado Blvd, Los Angeles, CA 90041

67. Lyndon Clarke, Edinburgh Parallel Computing Centre, James Clerk Maxwell Building, The King's Buildings, Mayfield Road, Edinburgh EH9 3JZ, United Kingdom

68. Michele Colajanni, Dip. di Ingegneria Elettronica, Universita' di Roma "Tor Vergata" Via della Ricerca Scientifica, 00133 - Roma, Italy

69. Jeremy Cook, Parallel Processing Laboratory, Dept. of Informatics, University of Bergen, High Technology Centre, N-5020 Bergen, Norway

70. Jim Cownie, Meiko Limited, 650 Aztec West, Bristol BS12 4SD, United Kingdom

71. Michel Dayde, CERFACS, 42 Avenue G. Coriolis, 31057 Toulouse Cedex, France

72. Mark Debbage, University of Southampton, Dept. of Electronics and Computer Science, Highfield, Southampton SO9 5NH, United Kingdom

73. John J. Dorning, Department of Nuclear Engineering Physics, Thornton Hall, McCormick Road, University of Virginia, Charlottesville, VA 22901

74. Tom Eidson, Theoretical Flow Physics Branch, M/S 156, NASA Langley Research Center, Hampton, VA 23665

75. Victor Eijkhout, University of Tennessee, 107 Ayres Hall, Department of Computer Science, Knoxville, TN 37996-1301

76. Anne Elster, Cornell University, Xerox DRI, 502 Engineering and Theory Center, Ithaca, NY 14853

77. Rob Falgout, Lawrence Livermore National Lab, L-419, P. O. Box 808, Livermore, CA 94551

78. Jim Feeney, IBM Endicott, R. D. 3, Box 224, Endicott, NY 13760 
79. Edward Felten, Department of Computer Science, lniversity of Washington, Seattle, WA 98195

80. Vince Fernando, NAG Limited, Wilkinson House, Jordan Hill Road, Oxford, OX2 8DR, United Kingdom

81. Randy Fischer, 615 NW 32st Place, Gainesville, FL 32607

82. Jon Flower, Parasoft Corporation, 2500 E. Foothill Blvd., Suite 205, Pasadena, CA91107

83. David Forslund, Los Alamos National Laboratory, Advanced Computing Laboratory, MS B287, Los Alamos, NM 87545

84. Geoffrey C. Fox, Syracuse University, Northeast Parallel Architectures Center 111 College Place, Syracuse, NY 13244-4100

85. Josef Fritscher, Computing Center, Technical University of Vienna, Wiedner Hauptstrasse 8-10, A-1040 Vienna, Austria

86. Daniel D. Frye, IBM Corporation, Dept. 49NA / MS 614, Neighborhood Road, Kingston, NY 12401

87. Kyle Gallivan, University of Illinois, CSRD, 465 CSRL, 1308 West Main Street, Urbana, IL 61801-2307

88. J. Alan George, Vice President, Academic and Provost, Needles Hall, University of Waterloo, Waterloo, Ontario, Canada N2L 3G1

89. Mike Gerndt, Zentralinstitut fuer Angewandte Mathematik, Forschungszentrum Juelich GmbH, Postfach 1913, D-5170 Juelich, Germany

90. Ian Glendinning, University of Southampton, Dept. of Electronics and Comp. Sci., Southampton, SO9 5NH, United Kingdom

91. Gene H. Golub, Department of Computer Science, Stanford University, Stanford, CA 94305

92. Adam Greenberg, Thinking Machines Corporation, 245 First Street, Cambridge, MA $02142-1214$

93. William Gropp, Argonne National Laboratory, Mathematics and Computer Science, 9700 South Cass Avenue, MCS 221, Argonne, IL 60439-4844

94. Sanjay Gupta, ICASE, Mail Stop 132C, NASA Langley Research Center, Hampton, VA 23665-5225

95. John Gustafson, 236 Wilhelm, Ames Laboratory, Iowa State University, Ames, IA 50011

96. Fred Gustavson, IBM T. J. Watson Research Center, Room 33-260, P. O. Box 218, Yorktown Heights, NY 10598

97. Robert Halstead, Digital Equipment Corporation, Cambridge Research Lab., One Kendall Sq. Bldg. 700, Cambridge, MA 02139

98. Robert J. Harrison, Battelle Pacific Northwest Laboratory, Mail Stop K1-90, P. O. Box 999, Richland, WA 99352

99. Leslie Hart, NOAA/FSL, R/E/FS5, 325 Broadway, Boulder, CO 80303 
100. Tom Haupt, Syracuse University, Northeast Parallel Architectures Center, 111 College Place, Syracuse, NY 13244-4100

101. Michael Heath, University of Illinois, NCSA, 4157 Beckman Institute, 405 North Mathews Avenue, Urbana, IL 61801-2300

102. Rolf Hempel, GMD, Schloss Birlinghoven, Postfach 13 16, D-W-5205 Sankt Augustin 1, Germany

103. Tom Henderson, NOAA/FSL, R/E/FS5, 325 Broadway, Boulder, CO 80303

104. Anthony J. G. Hey, University of Southampton, Dept. of Electronics and Comp. Sci., Southampton, SO9 5NH, United Kingdom

105. Mark Hill, University of Southampton, Dept. of Electronics and Comp. Sci., Southampton, $\mathrm{SO} 95 \mathrm{NH}$, United Kingdom

106. C. T. Howard Ho, IBM Almaden Research Center, K54/802, 650 Harry Road, San Jose, CA 95120

107. Steve Huss-Lederman, Supercomputing Research Center, 17100 Science Drive, Bowie, MD 20715-4300

108. S. Lennart Johnsson, Thinking Machines Corporation, 245 First Street, Cambridge, MA 02142-1214

109. Charles Jung, IBM Kingston, 67LB/MS 614, Neighborhood Road, Kingston, NY 12401

110. Malvyn H. Kalos, Cornell Theory Center, Engineering and Theory Center Bldg., Cornell University, Ithaca, NY 14853-3901

111. John Kapenga, Department of Computer Science, Western Michigan Universuty, Kalamazoo, MI 49008

112. Hans Kaper, Mathematics and Computer Science Division, Argonne National Laboratory, Bldg. 221, 9700 South Cass Avenue, Argonne, IL 60439

113. Udo Keller, PALlaS GmbH, Hermuelheimer Strasse 10, D-W5040 Bruehl, Germany

114. Ken Kennedy, Rice University, Department of Computer Science, P. O. Box 1892, Houston, TX 77251

115. Shlomo Kipnis, IBM T. J. Watson Research Center, PO Box 218, Yorktown Heights, NY 10598

116. Robert L. Knighten, Intel Corporation, Supercomputer Systems Division, 15201 NW Greenbrier Parkway, Beaverton, OR 97006

117. Charles Koelbel, Rice University, CITI/CRPC, P. O. Box 1892, Houston, TX 77251

118. Edward Kushner, Intel Corporation, 15201 NW Greenbrier Parkway, Beaverton, OR 97006

119. Falk Langhammer, Parsytec Computer GmbH, Juelicher Strasse 338, D-5100 Aachen, Germany

120. Randolph Langley, Florida State University, 400 SCL, B-186, Tallahassee, FL 32306 
121. Bob Leary, San Diego Supercomputer Center, P. O. Box 85608. San Diego, CA 92186-9784

122. Bruce Leasure, Kuck and Associates, Inc., 1906 Fox Drive, Champaign, IL 61820

123. James E. Leiss, Rt. 2, Box 142C, Broadway, VA 22815

124. John Lewis, Boeing Computer Services , Mail Stop 7L-21. P. O. Box 24346, Seattle, WA 98124-0346

125. Rik Littlefield, Battelle Pacific Northwest Laboratory, Mail Stop K1-87, P. O. Box 999, Richland, WA 99352

126. Miron Livny, University of Wisconsin, Department of Computer Science, 1210 West Dayton Street, Madison, WI 53706

127. Rusty Lusk, Argonne National Laboratory, Mathematics and Computer Science, 9700 South Cass Avenue, MCS 221, Argonne, IL 60439-4844

128. Arthur B. Maccabe, Sandia National Labs, Dept. 1424, Albuquerque, NM 871855800

129. Neil MacDonald, Edinburgh Parallel Computing Centre, James Clerk Maxwell Building, The King's Buildings, Mayfield Road, Edinburgh EH9 3JZ, United Kingdom

130. Peter Madams, nCUBE Corporation, 919 East Hillsdale Blvd., Foster City, CA 94404

131. David P. Mallon, Leeds University, School of Computer Studies, Leeds LS2 9JT, United Kir. gdom

132. Dan Cristian Marinescu, Computer Sciences Department, Purdue University, West Lafayette, IN 47907

133. Tim Mattson, Scientific Computing Associates, Inc., 265 Church Street, New Haven, CT 06510-7010

134. Oliver McBryan, University of Colorado at Boulder, Department of Computer Science, Campus Box 425, Boulder, CO 80309-0425

135. James McGraw, Lawrence Livermore National Laboratory, L-306, P. O. Box 808, Livermore, CA 94550

136. Phil McKinley, A714 Wells Hall, Michigan State University, East Lansing, MI 48824

137. Piyush Mehrotra, ICASE, Mail Stop 132C, NASA Langley Research Center, Hampton, VA 23665

138. Paul Messina, California Institute of Technology, Mail Stop 158-79, 1201 E. California Boulevard, Pasadena, CA 91125

139. Neville Moray, Department of Mechanical and Industrial Engineering, University of Illinois, 1206 West Green Street, Urbana, IL 61801

140. Charles Mosher, ARCO Exploration and Production Technology, 2300 West Plano Parkway, Plano, TX 75075-8499

141. Harish Nag, Intel Corporation, M/S CO4-02, 5200 Elam Young Parkway, Hillsboro, OR 97124 
142. Jonathan Nash, Leeds University, School of Computer Studies, Leeds LS2 9JT, United Kingdom

143. Dan Nessett, Lawrence Livermore National Laboratory, L-60, Livermore, CA 94550

144. Lionel M. Ni, Michigan State University, Dept. of Computer Science, A714 Wells Hall, East Lansing, MI 48824-102T

145. Mike Norman, Edinburgh Parallel Computing Centre, James Clerk Maxwell Building, The King's Buildings, Mayfield Road, Edinburgh, EH9 3JZ, United Kingdom

146. James M. Ortega, Department of Applied Mathematics, Thornton Hall, University of Virginia, Charlottesville, VA 22901

147. Steve Otto, Oregon Graduate Institute, Department of Computer Sci. and Eng., 19600 NW von Neumann Drive, Beaverton, OR 97006-1999

148. Andrea Overman, NASA Langley Research Center, MS 125, Hampton, VA 23665

149. Cherri M. Pancake, Department of Computer Science, Oregon State University, Corvallis, OR 97331-3202

150. Raj Panda, IBM Corporation, Mail Code E39/4305, 11400 Burnet Rd. , Austin, TX 78758

151. David Payne, Intel Corporation, Supercomputer Systems Division, 15201 NW Greenbrier Parkway, Beaverton, OR 97006

152. Arnulfo Perez, Centro de Intelligencia Artifical, ITESM, Suc. De Correos "J" C.P. 64849, Monterrey N.L., Mexico

153. Matthew Peters, Parallel and Distributed Processing, IBM UK Scientific Centre, Winchester, United Kingdom

154. Greg Pfister, IBM Corporation, Mail Stop 9462, 11400 Burnet Road, Austin, TX 78758-3493

155. Jean-Laurent Philippe, ARCHIPEL S.A., PAE des Glaisins, 1 rue du Bulloz, F74940 Annecy-le-Vieux, France

156. Paul Pierce, Intel Corporation, Supercomputer Systems Division, 15201 NW Greenbrier Parkway, Beaverton, OR 97006

157. Robert J. Plemmons, Departmen_- of Mathematics and Computer Science, Box 7311, Wake Forest University Winston-Salem, NC 27109

158. Steve Poole, 11631 Lima, Houston, TX 77099

159. Roldan Pozo, University of Tennessee, 107 Ayres Hall, Department of Computer Science, Knoxville, TN 37996-1301

160. Angela Quealy, Sverdrup Technology, Inc., NASA Lewis Research Center Group, 2001 Aerospace Pkwy, Brook Park, OH 44142

161. Padma Raghavan, University of Illinois, NCSA, 4151 Beckman Institute, 405 North Matthews Avenue, Urbana, IL 6180 .

162. Sanjay Ranka, Syracuse University, Northeast Parallel Architectures Center, 111 College Place, Syracuse, NY $1224: 4100$ 
163. Werner C. Rheinboldt, Department of Mathematics and Statistics, University of Pittsburgh, Pittsburgh, PA 15260

164. Peter Rigsbee, Cray Research Incorporated, 655 Lone Oak Drive, Eagan MN 55121

165. Matt Rosing, ICASE, Mail Stop 132C, NASA Langley Research Center, Hampton, VA $23665-5225$

166. Joel Saltz, ICASE, Mail Stop 132C, NASA Langley Research Center, Hampton, VA $23665-5225$

167. Ahmed H. Sameh, CSRD, University of Illinois 1308 West Main Street Urbana, IL $61801-2307$

168. Rob Schreiber, RIACS, Mail Stop T045-1, NASA Ames Research Center, Moffett Field, CA 94022

169. David S. Scott, Intel Scientific Computers, 15201 N. W. Greenbrier Parkway, Beaverton, OR 97006

170. Ambuj Singh, UC Santa Barbara, Department of Computer Science, Santa Barbara, CA 93106

171. Chuck Simmons, 500 Oracle Parkway, Box 659414, Redwood Shores, CA 94065

172. Anthony Skjellum, Mississippi State University, Department of Computer Science, Drawer CS, Mississippi State, MS 39762-5623

173. Steven G. Smith, Lawrence Livermore National Lab, L-419, P. O. Box 808, Livermore, CA 94550

174. Marc Snir, IBM T. J. Watson Research Center, PO Box 218, Room 28-226, Yorktown Heights, NY 10598

175. Karl Solchenbach, PALLAS GmbH, Hermuelheimer Strasse 10 D-5040 Bruehl Germany

176. Charles H. Still, Lawrence Livermore National Lab, L-416, P. O. Box 808, Livermore, CA 94550

177. Vaidy Sunderam, Emory University, Dept. of Math and Computer Science, Atlanta, GA 30322

178. Mike Surridge, Univ. of Southampton Parallel Applications Centre, 2 Venture Road, Chilworth Research Centre, Southampton SO1 7NP, United Kingdom

179. Alan Sussman, ICASE, Mail Stop 132C, NASA Langley Research Center, Hampton, VA 23665-5225

180. Paul N. Swartztrauber, National Center for Atmospheric Research, P. O. Box 3000, Boulder, CO 80307

181. Clemens-August Thole, GMD-I1.T, Schloss Birlinghoven, D-5205 Sankt Augustin 1, Germany

182. Anne Trefethen, Engineering and Theory Center, Cornell University, Ithaca, NY 14853

183. Christian Tricot, ARCHIPEL S.A., PAE des Glaisins, 1 rue du Bulloz, F-74940 Annecy-le-Vieux, France 
184. Anna Tsao, Supercomputing Research Center, 17100 Science Drive, Bowie, MD 20715-4300

185. Lew Tucker, Thinking Machines Corporation, 245 First Street, Cambridge, MA 02142-1214

186. Robert van de Geijn, University of 'Texas, Department of Computer Sciences, TAI 2.124, Austin, TX 78712

187. Robert G. Voigt, National Science Foundation, Room 417, 1800 G Street, N.W., Washington, DC 20550

188. Linton Ward, 11400 Burnet Rd, Austin, TX 78758

189. Dennis Weeks, Convex Computer Corp., 3000 Waterview Parkway, P. O. Box 833851, Richardson, TX 75081

190. Tammy Welcome, Lawrence Livermore National Lab, Massively Parallel Computing Initiative, L-416, P. O. Box 808, Livermore, CA 94550

191. Jim West, IBM Corporation, MC 5600, 3700 Bay Area Blvd., Houston, TX 77058

192. Stephen R. Wheat, Dept. 1424, Sandia National Labs, Albuquerque, NM 871855800

193. Mary F. Wheeler, Rice University, Department of Mathematical Sciences, P. O. Box 1892, Houston, TX 77251

194. Andrew B. White, Computing Division, Los Alamos National Laboratory, P. O. Box 1663, MS-265, Los Alamos, NM 87545

195. Joel Williamson, Convex Computer Corporation, 3000 Waterview Parkway, Richardson, TX 75083-3851

196. Steve Zenith, Kuck and Associates, Inc., 1906 Fox Drive, Champaign, IL 618207334

197. Mohammad Zubair, NASA Langley Research Center, Mail Stop 132C, Hampton, VA 23665

198. Office of Assistant Manager for Energy Research and Development, U.S. Department of Energy, Oak Ridge Operations Office, P. O. Box 2001 Oak Ridge, 'TN $37831-8600$

199-208. Office of Scientific \& Technical Information, P. O. Box 62, Oak Ridge, TN 37831 

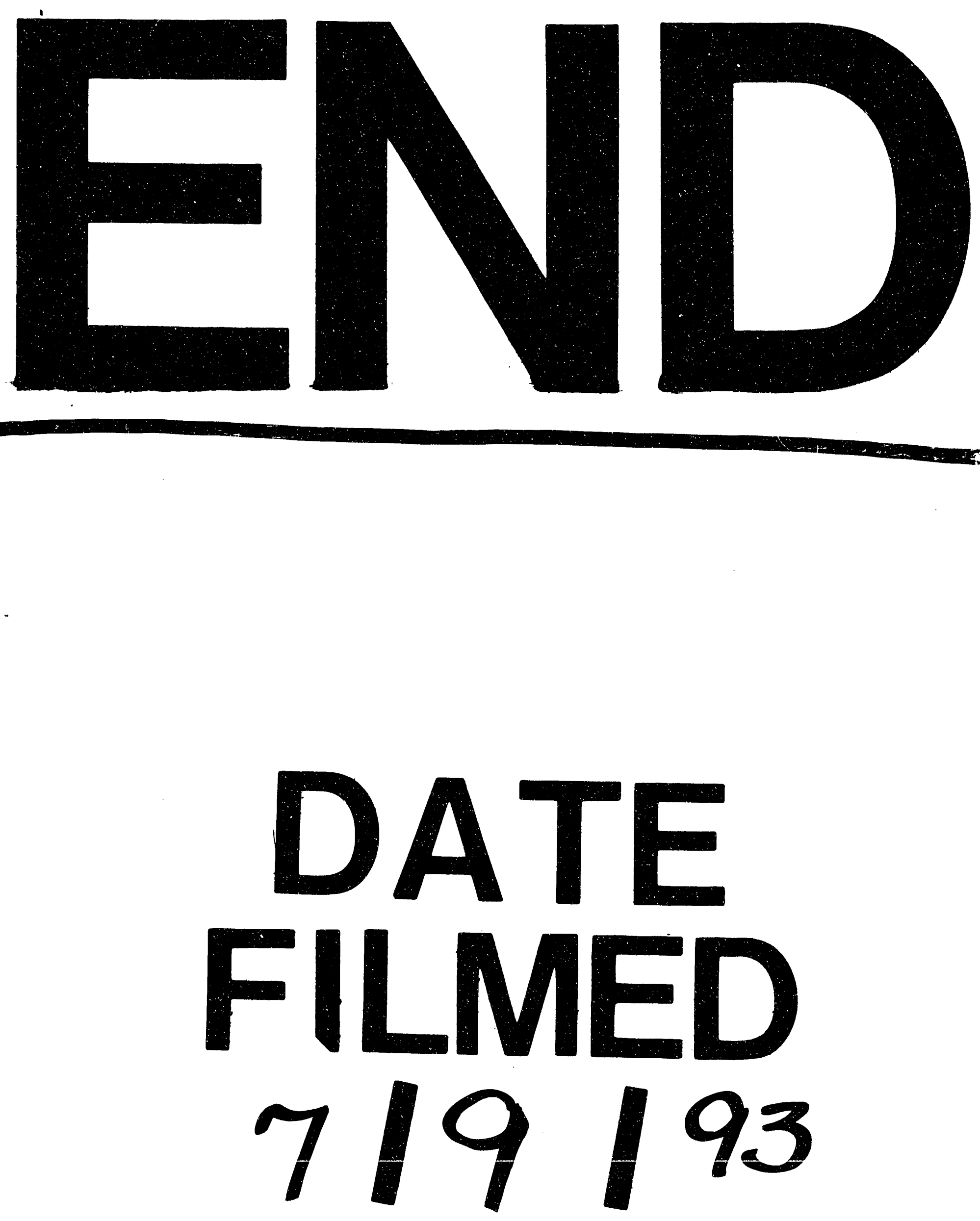
